\title{
Gauge invariant effective action for the Polyakov line in the SU(N) Yang-Mills theory at high temperatures
}

\author{
Dmitri Diakonov ${ }^{a, \text {, }}$ and Michaela Oswald \\ ${ }^{a}$ NORDITA, Blegdamsvej 17, DK-2100 Copenhagen, Denmark \\ ${ }^{b}$ St. Petersburg NPI, Gatchina, 188 300, St. Petersburg, Russia \\ ${ }^{c}$ NBI, Blegdamsvej 17, DK-2100 Copenhagen, Denmark
}

(Dated: September 14, 2004)

\begin{abstract}
We integrate out fast varying quantum fluctuations around static $A_{4}$ and $A_{i}$ fields for the $S U(N)$ gauge group. By assuming that the gluon fields are slowly varying but allowing for an arbitrary amplitude of $A_{4}$ we obtain two variants of the effective high-temperature theory for the Polyakov line. One is the effective action for the gauge-invariant eigenvalues of the Polyakov line, and it is explicitly $Z(N)$ symmetric. The other is the effective action for the Polyakov line itself as an element of the $S U(N)$. In this case the theory necessarily includes the spatial components $A_{i}$ to ensure its gauge invariance under spatial gauge transformations. We derive the 1-loop effective action in the 'electric' and 'magnetic' sectors, summing up all powers of $A_{4}$.
\end{abstract}

PACS numbers: 11.15.-q,11.10.Wx,11.15.Tk

Keywords: gauge theories, finite temperature field theory, derivative expansion

\section{INTRODUCTION}

Finite-temperature quantum chromodynamics (QCD) is an intensely studied field. At finite temperature gluons obey periodic boundary conditions in imaginary time, leading to the quantized Matsubara frequencies $\omega_{k}=$ $2 \pi k T$ for gluons. At very high temperatures nonzero Matsubara frequencies decouple from a theory as they become infinitely heavy. This is called dimensional reduction [1] since the decoupled heavy modes are timedependent ones. Neglecting all modes except the static zero Matsubara frequencies leaves a 3D theory

$$
-\frac{1}{4 g^{2}} \int d^{4} x F_{\mu \nu}^{2} \rightarrow-\frac{1}{4 g^{2} T} \int d^{3} x\left[F_{i j}^{2}+2\left(D_{i}^{a b} A_{4}^{b}\right)^{2}\right]
$$

which only contains the static gluonic modes with the coupling constant $g_{3}^{2}=g^{2} T$. The long-range forces mediated by the static gluons lead to the IR divergencies, because in strict perturbation theory the gluons are massless. Owing to the chromomagnetic sector, the hightemperature perturbation theory explodes already at a few-loop level [2, 3, 4] and is hence only applicable at academically high temperatures [5]. The region of intermediate temperatures is of much bigger interest. For example, the deconfinement phase transition in the pure Yang-Mills theory is believed to take place in this region.

In the pure Yang-Mills theory the center symmetry plays a crucial part in the description of the confinementdeconfinement phase transition [2, 6, 7, 8]. The order parameter for the latter is the average of the trace of the

\footnotetext{
*Electronic address: diakonov@nordita.dk
}

${ }^{\dagger}$ Electronic address: oswald@alf.nbi.dk
Polyakov line:

$$
L(x)=\mathcal{P} \exp \left(i \int_{0}^{1 / T} d x_{4} A_{4}\right) .
$$

The order parameter $\langle\operatorname{Tr} L\rangle$ is zero in the confined phase below the critical temperature and assumes, after a proper regularization, a non-zero value in the deconfined phase above the critical temperature. For a recent confirmation in lattice simulations see [9, 10]. The Polyakov line is not invariant under gauge transformations belonging to the gauge group center. One hence concludes that if $\langle\operatorname{Tr} L>=0$ then the $Z(N)$ symmetry is manifest. This situation describes confinement. If for any reason $<\operatorname{Tr} L>\neq 0$ then the symmetry must have been broken. This corresponds to the deconfined phase.

At high but not infinitely high temperatures the treelevel action (11) is not sufficient to study gluon fluctuations. When one includes quantum corrections all the non-zero Matsubara modes of the gluons show up in the loops. The 1-loop [4, 11] and 2-loop 12 potential energies as functions of $A_{4}$ are known. They are periodic functions of the eigenvalues of $A_{4}$ in the adjoint representation with period $2 \pi T$. This reflects the symmetry of the $Z(N)$ vacua. The curvature of the potential at its minima gives the leading order Debye mass for 'electric' gluons. The zero energy minima of the potential are at quantized values of $A_{4}$ corresponding to the centerof-group values of the Polyakov line, where $L \in Z(N)$. At high temperatures the system oscillates around one of these minima. At lower temperatures, however, the fluctuations around the minimum increase and eventually the system undergoes a phase transition to $\langle\operatorname{Tr} L\rangle=0$. At the same time, one expects that near the phase transition point the fluctuations are long-range. To study those fluctuations, one needs an effective low-momenta theory which, however, does not assume that the $A_{4}$ component is small. 
The nonzero temperatures explicitly break the $4 \mathrm{D} \mathrm{Eu-}$ clidean symmetry of the theory down to the 3D Euclidean symmetry, so that the spatial $A_{i}$ and time $A_{4}$ components of the Yang-Mills field play different roles and should be treated differently. One can always choose a gauge where $A_{4}$ is time-independent. Taking $A_{4}(x)$ to be static is not a restriction of any kind on the fields but merely a convenient gauge choice, and we shall imply this gauge throughout the paper. It is also a possible gauge choice at $T=0$ but in that limiting case it is unnatural as one usually wishes to preserve the $4 \mathrm{D}$ symmetry. In this gauge, the Polyakov line (21) is not a path-ordered but a simple exponent of $A_{4}(x)$. It rotates under $x$-dependent gauge transformations. Therefore, an effective action for the Polyakov line, which should be invariant under spatial gauge transformations, cannot depend on the gradients of $L(x)$ alone, but rather on the covariant derivatives of $L(x)$, involving the spatial components of the background field $A_{i}$. The aim of this work is to compute the effective action expanding it in covariant derivatives but keeping all powers of $A_{4}$. It is an extension of the previous work 13, 14, 15] to higher gauge groups. We use the background field method for the gluons and evaluate the 1-loop action through the functional determinant formalism. We integrate out fast varying quantum fluctuations about the static background $\left(A_{i}, A_{4}\right)$ by making an expansion in spatial covariant derivatives. This method was originally developed in [16] for zero temperature QCD. We compute terms quadratic in covariant derivatives, that is the 'electric' $E^{2}$ terms where $E_{i}^{a}=D_{i}^{a b} A_{4}^{b}$, and the quartic 'magnetic' $B_{\|}^{2}$ terms where $B_{i}^{a}=\frac{1}{2} \epsilon_{i j k}\left(\partial_{i} A_{j}^{a}-\partial_{j} A_{i}^{a}+f^{a b c} A_{i}^{b} A_{j}^{c}\right)$. While we consider a general electric field, we restrict ourselves to a magnetic field parallel to $A_{4}, B_{i}=B_{i \|}$. The structure of the $S U(3)$ effective action turns out to be much richer than in the $S U(2)$ case, as there are far more invariants which one can build out of $A_{4}$ and the electric and the magnetic fields.

As a byproduct of our study, we obtain the effective action for the eigenvalues of the Polyakov line. Contrary to the Polyakov line itself which is an element of the $S U(N)$ group and rotates under spatial gauge transformations, its eigenvalues are gauge invariant. Therefore, the derivative expansion of the effective action for the eigenvalues goes in ordinary rather than covariant derivatives. Such an action was first obtained in ref. [17] for the $S U(2)$ gauge group and reaffirmed in ref. [13]; here we extend it first to the more intricate case of the $S U(3)$ gauge group and then to the general $S U(N)$. The resulting action is explicitly $Z(N)$ symmetric.

We expect that our results are suitable to study the correlation functions of the Polyakov line not too far from the transition point where it experiences fluctuations that are large in amplitude but presumably mainly long ranged.

\section{COVARIANT DERIVATIVE EXPANSION OF 1-LOOP QUANTUM ACTION}

In 13] we obtained the 1-loop effective action for the pure Yang-Mills $S U(2)$ theory. We started with the Yang-Mills partition function and decomposed the gluon fields into background fields and quantum fluctuations around them. For a 1-loop approximation we expanded the action to quadratic order in the gluonic quantum fluctuations. The resulting bilinear form for the quantum fluctuations is degenerate, so we chose a background Lorenz gauge for them. This in turn gives rise to the Faddeev-Popov ghost determinant in the background field. The 1-loop effective action was obtained by integrating over the ghost fields as well as over the gluon quantum fluctuations. It is expressed in terms of functional determinants which is an economic and aesthetic method of getting gauge invariant results 24]:

$$
S_{1-\text { loop }}=\log (\operatorname{det} W)^{-1 / 2}+\log \operatorname{det}\left(-D^{2}\right) .
$$

The first contribution here comes from integrating out the gluon fields, while the second comes from the ghost fields. Since the gluons transform under the adjoint representation of the color group the operators $D^{2}, W_{\mu \nu}$ consist of matrices in the adjoint representation. The latter is given by

$$
W_{\mu \nu}^{a b}=-\left[D^{2}(A)\right]^{a b} \delta_{\mu \nu}-2 f^{a c b} F_{\mu \nu}^{c}(A),
$$

where

$$
D_{\mu}^{a b}=\partial_{\mu} \delta^{a b}+f^{a c b} A_{\mu}^{c}
$$

is the covariant derivative in the adjoint representation. All gluon fields $A_{\mu}$ are background fields. Eq. (3) is independent of the gauge group and hence valid for any $S U(N)$. In addition, it is invariant under general gauge transformations of the $A_{\mu}$ fields. We can use this property to make the $A_{4}$ component static. Then, there still remains a freedom to make time-independent gauge transformations,

$$
\begin{aligned}
& A_{4}(x) \rightarrow U(x) A_{4}(x) U^{\dagger}(x), \\
& A_{i}(x) \rightarrow U(x) A_{i}(x) U^{\dagger}(x)+i U(x) \partial_{i} U^{\dagger}(x),
\end{aligned}
$$

which can be used to bring $A_{4}$ to a diagonal form in the fundamental representation. The general $S U(N)$ case is considered in section XI,B. For the $S U(3)$ gauge group we can write ( $\lambda^{a}$ are 8 Gell-Mann matrices, see the Appendix)

$$
A_{4}=A_{4}^{3}(x) \frac{\lambda^{3}}{2}+A_{4}^{8}(x) \frac{\lambda^{8}}{2} .
$$

This gauge fixing leaves $A_{i}$ an arbitrary $S U(3)$ matrix, generally speaking both space and time dependent.

After fixing the gauge such that $A_{4}$ is diagonal and static, one is still left with a freedom of gauge transformations of a special type. For the $S U(2)$ group they were 
pointed out in ref. [13], section 10; in the $S U(3)$ case the remaining gauge transformations are of the form

$$
\begin{aligned}
A_{\mu} & \rightarrow S A_{\mu} S^{\dagger}+i S \partial_{\mu} S^{\dagger} \\
S(x, t) & =\exp \left\{i \frac{\lambda^{3}}{2}\left[\alpha_{3}(x)+2 \pi t T n_{3}\right]+i \frac{\lambda^{8}}{2}\left[\alpha_{8}(x)+2 \pi t T n_{8}\right]\right\}
\end{aligned}
$$

where $n_{3}$ and $n_{8} \sqrt{3}$ are both even or both odd integers. One cannot take rotations about axes other than the $3^{\text {rd }}$ and $8^{\text {th }}$ ones because it will make $A_{4}$ non-diagonal, and one cannot take the time dependence other than linear because that would make $A_{4}$ time dependent. The spacedependent functions $\alpha_{3,8}$ may be arbitrary. The fact that $n_{3}$ and $n_{8} \sqrt{3}$ must be both even or both odd integers follows from the requirement that the gauge transformation in the adjoint representation, given by the $8 \times 8$ matrix $O^{a b}=2 \operatorname{Tr}\left(S t^{a} S^{\dagger} t^{b}\right)$, must be periodic in time. In components, the transformation (8) reads:

$$
\begin{aligned}
A_{4}^{3,8} & \rightarrow A_{4}^{3,8}+2 \pi T n_{3,8}, \\
A_{i}^{3,8} & \rightarrow A_{i}^{3,8}+\partial_{i} \alpha_{3,8}, \\
A_{i}^{1} & \rightarrow \cos \beta_{3} A_{i}^{1}+\sin \beta_{3} A_{i}^{2}, \\
A_{i}^{2} & \rightarrow-\sin \beta_{3} A_{i}^{1}+\cos \beta_{3} A_{i}^{2}, \\
A_{i}^{4} & \rightarrow \cos \frac{\beta_{8}+\beta_{3}}{2} A_{i}^{4}+\sin \frac{\beta_{8}+\beta_{3}}{2} A_{i}^{5}, \\
A_{i}^{5} & \rightarrow-\sin \frac{\beta_{8}+\beta_{3}}{2} A_{i}^{4}+\cos \frac{\beta_{8}+\beta_{3}}{2} A_{i}^{5}, \\
A_{i}^{6} & \rightarrow \cos \frac{\beta_{8}-\beta_{3}}{2} A_{i}^{6}+\sin \frac{\beta_{8}-\beta_{3}}{2} A_{i}^{7}, \\
A_{i}^{7} & \rightarrow-\sin \frac{\beta_{8}-\beta_{3}}{2} A_{i}^{6}+\cos \frac{\beta_{8}-\beta_{3}}{2} A_{i}^{7}, \\
\beta_{3,8} & =2 \pi t T n_{3,8}+\alpha_{3,8} .
\end{aligned}
$$

The Polyakov line is transformed by a diagonal matrix

$$
\operatorname{diag}\left(e^{\pi i\left(n_{3}+\frac{n_{8}}{\sqrt{3}}\right)}, e^{-\pi i\left(n_{3}-\frac{n_{8}}{\sqrt{3}}\right)}, e^{-\frac{2 \pi i}{\sqrt{3}} n_{8}}\right),
$$

which for $n_{3}$ and $n_{8} \sqrt{3}$ both even or both odd becomes an element of the group center, $\operatorname{diag}\left(e^{\frac{2 \pi i k}{3}}, e^{\frac{2 \pi i k}{3}}, e^{\frac{2 \pi i k}{3}}\right)$, $k=0,1,2$.

Thus we see that the $Z(N)$ symmetry of the effective action is a consequence of the symmetry under gauge transformations of a special type, eq. (8). It should be stressed, however, that the same gauge transformations make the spatial components of the background field $A_{i}$ time dependent, even if one starts from purely static $A_{i}$ 's, with their Matsubara frequencies directly related to $n_{3,8}$ according to eqs. (1116). Consequently, if one wishes to see the $Z(N)$ symmetry of the effective action (3) explicitly, one has to sum up all powers in time derivatives of $A_{i}$. This seems to be a formidable problem which we do not attempt to solve here. We expand the effective action (3) to the second order in the electric field and hence to the second order in $\dot{A}_{i}$ only. Therefore, our effective action will not be explicitly $Z(N)$ symmetric. To be more precise, part of the invariants we calculate will be explicitly $Z(N)$ symmetric, namely those which remain invariant under the transformations (1116), but some of the invariants will be not. The effective action we are computing is for the static $A_{i}$ fields and is invariant only under static gauge transformations (6).

With our background-field technique, we are also able to solve simultaneously another physical problem. Namely, one may be interested in the effective quantum action for the eigenvalues of the Polyakov line. Contrary to $A_{4}$ and to the Polyakov line as a unitary matrix, its eigenvalues are invariant under spatial gauge transformations. Therefore, the effective action for the eigenvalues can be expanded in ordinary 'short' rather than covariant derivatives, and the background $A_{i}$ field may then be set to zero. In this setting, the $A_{i}$ fields (as well as the rapidly changing components of the $A_{4}$ fields) are understood and treated as quantum fluctuations over which one integrates. The resulting effective action for the eigenvalues of the Polyakov line, including its spatial derivatives to the second order, explicitly obeys the $Z(N)$ symmetry. It has been first found for the $S U(2)$ gauge group in ref. 17] and reaffirmed in ref. [13]; here we extend this result to the $S U(3)$ case in section XI,A and to the general $S U(N)$ gauge group in section XI,B.

The functional determinants in eq. (3) are UV divergent which reflects the divergence of the coupling constant. Since QCD is a renormalizable theory the divergence can be absorbed in the definition of the tree level coupling constant. To do so, we have to properly normalize and regularize the functional determinants. The former is obtained by normalizing to the free zero gluon contribution, for the latter we introduce a Pauli-Villars cutoff $M$ in momentum space. For the gluon determinant this results in the "quadrupole formula"

$$
\begin{gathered}
\operatorname{det}\left(-D^{2}\right)_{\mathrm{r}, \mathrm{n}} \equiv \frac{\operatorname{det}\left(-D_{\mu}^{2}\right)}{\operatorname{det}\left(-\partial_{\mu}^{2}\right)} \frac{\operatorname{det}\left(-\partial_{\mu}^{2}+M^{2}\right)}{\operatorname{det}\left(-D_{\mu}^{2}+M^{2}\right)} \\
\quad=\exp \left\{-\int_{0}^{\infty} \frac{d s}{s} \operatorname{Sp}\left[\left(1-e^{-s M^{2}}\right)\left(e^{s D_{\mu}^{2}}-e^{s \partial_{\mu}^{2}}\right)\right]\right\}
\end{gathered}
$$

where the rewriting in the last line is due to a trick originally introduced by Schwinger [18]. The functional trace Sp can be taken by inserting any complete basis. We choose the plane wave basis:

$$
\begin{aligned}
\operatorname{Sp} e^{-s K} & =\operatorname{Tr} \int d^{4} x \lim _{y \rightarrow x} \int \frac{d^{4} p}{(2 \pi)^{4}} e^{-i p \cdot y} e^{-s K} e^{i p \cdot x} \\
& =\operatorname{Tr} \int d^{4} x \int \frac{d^{4} p}{(2 \pi)^{4}} e^{-s K\left(\partial_{\alpha} \rightarrow \partial_{\alpha}+i p_{\alpha}\right)} \mathbf{1} .
\end{aligned}
$$

Here $\operatorname{Tr}$ is the remaining trace over color and Lorentz indices. The $\mathbf{1}$ at the end is meant to emphasize that the shifted operator acts on unity, so that for example any term that has a $\partial_{\alpha}$ in the exponent and is brought all the way to the right, will vanish. According to (20) we now 
have

$$
\begin{aligned}
& \log \operatorname{det}\left(-D^{2}\right)_{\mathrm{r}, \mathrm{n}}= \\
& \quad-\int d^{3} x \sum_{k=-\infty}^{\infty} \int \frac{d^{3} p}{(2 \pi)^{3}} \int_{0}^{\infty} \frac{d s}{s}\left(1-e^{-s M^{2}}\right) e^{-s p^{2}} \\
& \quad \times \operatorname{Tr}\left\{\exp \left[s \mathcal{A}^{2}+s D_{i}^{2}+2 i s p_{i} D_{i}\right]-\exp \left[-s \omega_{k}^{2}\right]\right\}
\end{aligned}
$$

where we introduced the adjoint matrix

$$
\mathcal{A}=f^{a c b} A_{4}^{c}+i \omega_{k} \delta^{a b} .
$$

Similarly to eq. (19) we have to normalize and regularize the gluon functional determinant. Again after an insertion of a plane wave basis we obtain

$$
\begin{aligned}
& \log (\operatorname{det} W)_{\mathrm{r}, \mathrm{n}}^{-1 / 2}= \\
& \frac{1}{2} \int d^{3} x \sum_{k=-\infty}^{\infty} \int \frac{d^{3} p}{(2 \pi)^{3}} \int_{0}^{\infty} \frac{d s}{s}\left(1-e^{-s M^{2}}\right) e^{-s p^{2}} \\
& \times \operatorname{Tr}\left\{\exp \left[\left(s \mathcal{A}^{2}+s D_{i}^{2}+2 i s p_{i} D_{i}\right)^{a b} \delta_{\mu \nu}+2 s f^{a c b} F_{\mu \nu}^{c}\right]\right. \\
& \left.\quad-\exp \left[-s \omega_{k}^{2}\right]\right\} .
\end{aligned}
$$

The regularized and normalized effective 1-loop action is hence given by the sum of eq. (21) and eq. (23). Expressed in this way one can now expand in powers of spatial covariant derivatives $D_{i}$. This introduces a potential energy and a kinetic energy in terms of color-electric and color-magnetic fields, since the latter are identified as

$$
\begin{aligned}
i\left[D_{i}, \mathcal{A}\right] & =i\left[D_{i}, D_{4}\right]=F_{i 4}=E_{i} \quad \text { and } \\
\frac{1}{2} \epsilon_{i j k} F_{j k} & =\frac{i}{2}\left[D_{j}, D_{k}\right]=B_{i} .
\end{aligned}
$$

In 13] we studied the effective action for the gauge group $S U(2)$. We expanded to quadratic order in the electric and magnetic fields, but did not retain mixing terms between electric and magnetic field or derivative terms of the electric field, which all exist at that order. The gauge invariant structures that we obtained were $A_{4}^{a} A_{4}^{a}, E_{i}^{a} E_{i}^{a}$, $B_{i}^{a} B_{i}^{a},\left(E_{i}^{a} A_{4}^{a}\right)^{2}$ and $\left(B_{i}^{a} A_{4}^{a}\right)^{2}$. For $S U(3)$, which is the case that we are considering here, one expects and finds more invariants.

\section{THE POLYAKOV LINE}

The order parameter for the transition from a confined to a deconfined phase in a pure Yang-Mills theory is the average of the trace of the Polyakov line,

$$
L(x)=\mathcal{P} \exp \left\{i \int_{0}^{\beta=\frac{1}{T}} d x_{4} A_{4}\left(x_{4}, x\right)\right\} .
$$

As usual $\mathcal{P}$ denotes path ordering. For our explicit calculations we shall use the freedom to rotate the $A_{4}$ field to a diagonal form and consider it to be static. In this gauge, $L(x)$ is a diagonal matrix $\exp \left(i A_{4} / T\right)$. For an
$S U(N)$ there are $(N-1)$ diagonal generators. In $S U(2)$ this is the third Pauli matrix $\tau_{3}$, in $S U(3)$ one has the $\lambda^{3}$ and $\lambda^{8}$ Gell-Mann matrices (the general $S U(N)$ case will be considered in section XIB). Writing $A_{4}$ in the basis of these diagonal generators automatically ensures that it is traceless.

In particular we choose the following parametrization $\left(t^{a}=\lambda^{a} / 2\right.$, see Appendix)

$$
A_{4}=A_{4}^{3} t^{3}+A_{4}^{8} t^{8} .
$$

From this we can then construct $A_{4}$ in the adjoint representation as $A_{4}^{\text {adj }}=A_{4}^{a b}=i f^{a c b} A_{4}^{c}$. We find that it has the following non-zero eigenvalues

$$
\begin{aligned}
& \pm \phi_{1}= \pm A_{4}^{3}, \\
& \pm \phi_{2}= \pm \frac{A_{4}^{3}+A_{4}^{8} \sqrt{3}}{2}, \\
& \pm \phi_{3}= \pm \frac{A_{4}^{3}-A_{4}^{8} \sqrt{3}}{2} .
\end{aligned}
$$

Introducing the rescaled variables $a_{3} \equiv A_{4}^{3} /(2 \pi T), a_{8} \equiv$ $A_{4}^{8} /(2 \pi T)$ and $\nu_{i}=\phi_{i} /(2 \pi T)$, eq. (27) becomes

$$
\begin{aligned}
& \pm \nu_{1}= \pm a_{3}, \\
& \pm \nu_{2}= \pm \frac{a_{3}+a_{8} \sqrt{3}}{2}, \\
& \pm \nu_{3}= \pm \frac{a_{3}-a_{8} \sqrt{3}}{2} .
\end{aligned}
$$

The Polyakov line $L=\exp \left(i A_{4} / T\right)$ is a diagonal unitary matrix:

$$
\begin{aligned}
L & =\left(\begin{array}{ccc}
e^{\pi i\left(a_{3}+\frac{a_{8}}{\sqrt{3}}\right)} & 0 & 0 \\
0 & e^{-\pi i\left(a_{3}-\frac{a_{8}}{\sqrt{3}}\right)} & 0 \\
0 & 0 & e^{-\frac{2 \pi i}{\sqrt{3}} a_{8}}
\end{array}\right) \\
& =\left(\begin{array}{ccc}
e^{\frac{2 \pi i}{3}\left(\nu_{1}+\nu_{2}\right)} & 0 & 0 \\
0 & e^{-\frac{2 \pi i}{3}\left(\nu_{1}+\nu_{3}\right)} & 0 \\
0 & 0 & e^{-\frac{2 \pi i}{3}\left(\nu_{2}-\nu_{3}\right)}
\end{array}\right) .
\end{aligned}
$$

Here we expressed it once in terms of $a_{3}$ and $a_{8}$ and once through the eigenvalues of $A_{4}^{\text {adj }}$. It is easy to see that the Polyakov line (29) assumes values of the group center, $e^{2 \pi i k / 3} \mathbf{1}_{3}, k=0,1,2$, for integer values of $\nu_{i}$. This knowledge will be of use to understand the potential energy in the next section.

\section{THE POTENTIAL ENERGY}

The potential energy is the contribution to the 1-loop effective action that corresponds to constant background fields, hence all spatial variations are set to zero. Since in Yang-Mills theories gauge invariance requires that derivatives always show up in their covariant form, we obtain the potential by setting $D_{i}$ equal to zero. From 
eq. (3) and eqs. 21] 23) we see that $F_{\mu \nu}$ does not contribute since it is either linear $\left(F_{4 i}\right)$ or quadratic $\left(F_{i j}\right)$ in the covariant derivatives. Therefore, we find that at zeroth order in $D_{i}$

$$
\begin{aligned}
S_{1-\text { loop }}^{(0)} & =-\left[\log \operatorname{det}\left(-D^{2}\right)\right]^{(0)}= \\
& =\int d^{3} x \sum e^{-s p^{2}} \operatorname{Tr}\left(e^{-s \mathcal{A}^{2}}-e^{-s \omega_{k}^{2}}\right) \\
& =-\int d^{3} x P\left(A_{4}\right) .
\end{aligned}
$$

Here we introduce compact notations:

$$
\begin{aligned}
\mathcal{Y} & =\sum_{k=-\infty}^{\infty} \int \frac{d^{3} p}{(2 \pi)^{3}} \int_{0}^{\infty} \frac{d s}{s} \\
\mathcal{Y}^{M} & =\sum_{k=-\infty}^{\infty} \int \frac{d^{3} p}{(2 \pi)^{3}} \int_{0}^{\infty} \frac{d s}{s}\left(1-e^{-s M^{2}}\right) .
\end{aligned}
$$

For eq. (30) we need the matrix $\mathcal{A}^{a b}$. With the Ansatz (26) we can construct and express it in terms of the eigenvalues of $A_{4}^{\text {adj }}$, eq. (27):

$$
\mathcal{A}=\left(\begin{array}{cccccccc}
i \omega_{k} & -\phi_{1} & 0 & 0 & 0 & 0 & 0 & 0 \\
\phi_{1} & i \omega_{k} & 0 & 0 & 0 & 0 & 0 & 0 \\
0 & 0 & i \omega_{k} & 0 & 0 & 0 & 0 & 0 \\
0 & 0 & 0 & i \omega_{k} & -\phi_{2} & 0 & 0 & 0 \\
0 & 0 & 0 & \phi_{2} & \omega_{k} & 0 & 0 & 0 \\
0 & 0 & 0 & 0 & 0 & i \omega_{k} & \phi_{3} & 0 \\
0 & 0 & 0 & 0 & 0 & -\phi_{3} & \omega_{k} & 0 \\
0 & 0 & 0 & 0 & 0 & 0 & 0 & i \omega_{k}
\end{array}\right)
$$

We then find that

$$
\begin{aligned}
& \operatorname{Tr}\left[e^{-s \mathcal{A}^{2}}-e^{-s \omega_{k}^{2}}\right] \\
= & \sum_{n=1,2,3}\left[\left(e^{-s\left(\phi_{n}-\omega_{k}\right)^{2}}+e^{-s\left(\phi_{n}+\omega_{k}\right)^{2}}\right)-2 e^{-s \omega_{k}^{2}}\right]
\end{aligned}
$$

where the sum is over the adjoint eigenvalues. In the $S U(2)$ case there is just one term yielding [13]

$$
\begin{aligned}
& 2 \sum e^{-s p^{2}}\left[e^{-s\left(\phi-\omega_{k}\right)^{2}}-e^{-s \omega_{k}^{2}}\right] \\
= & -\left.\frac{1}{12 \pi^{2} T} \phi^{2}(2 \pi T-\phi)^{2}\right|_{\bmod 2 \pi T .}
\end{aligned}
$$

In the $S U(3)$ case one obtains [4]

$$
P\left(A_{4}\right)=\left.\frac{(2 \pi)^{2} T^{3}}{3} \sum_{n=1,2,3} \nu_{n}^{2}\left(1-\nu_{n}\right)^{2}\right|_{\bmod 1},
$$

where again

$$
\nu_{1}=\frac{A_{4}^{3}}{2 \pi T}, \quad \nu_{2}=\frac{1}{2} \frac{A_{4}^{3}+A_{4}^{8} \sqrt{3}}{2 \pi T}, \quad \nu_{3}=\frac{1}{2} \frac{A_{4}^{3}-A_{4}^{8} \sqrt{3}}{2 \pi T} .
$$

We hence see that the potential energy is periodic in the eigenvalues of $A_{4}^{\text {adj }}$ with period $2 \pi T$. We plot it as

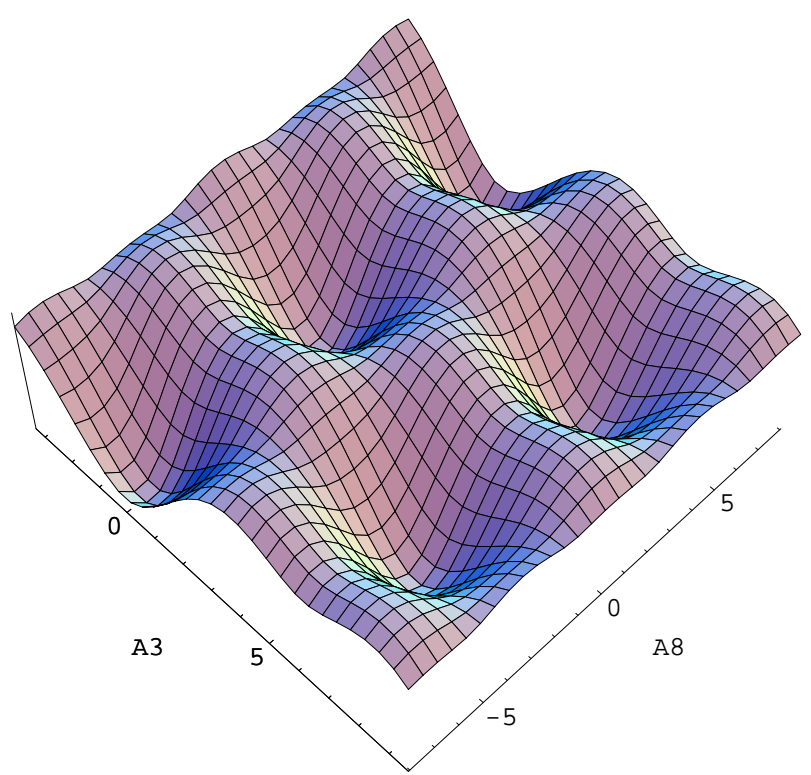

FIG. 1: Potential energy as function of $A_{4}^{3}$ and $A_{4}^{8}$ forms a double-periodic triangle lattice. The $Z(3)$ symmetry is the symmetry under lattice translations.

a function of $A_{4}^{3}$ and $A_{4}^{8}$ in Fig. 1. In these axes, the minima and maxima of the potential energy form a regular triangle lattice, with lattice spacing $2 \pi T$. The zeroenergy minima are at integer values of $\nu_{n}, n=1,2,3$, where the Polyakov line assumes values from the group center. The maxima of the potential energy correspond to the Polyakov line $L_{\max }=\operatorname{diag}\left(1, e^{\frac{2 \pi i}{3}}, e^{-\frac{2 \pi i}{3}}\right)$ (and those obtained from this one by multiplying it by the elements of the group center) whose $\operatorname{Tr} L_{\max }=0$. At high temperatures $L(x)$ oscillates somewhat around the trivial center-of-group minima of the potential. However, as one lowers the temperature the fluctuations of the Polyakov line around these perturbative minima become stronger and eventually a transition to a phase with $\langle\operatorname{Tr} L\rangle=0$ occurs, despite the fact that the potential energy is maximal at $\operatorname{Tr} L=0$. The aim of the rest of the paper is to derive the effective action for the fluctuations of $L(x)$ in a broad range of its variation beyond the trivial minima.

\section{THE ELECTRIC SECTOR}

As in 13] we are interested in the leading terms for the kinetic energy in the color-electric sector, meaning terms quadratic in the electric field. To obtain these, we have to expand eqs. 211 231) to quadratic order in $D_{i}$, because the electric field is identified as eq. (24). Since $D_{i}$ shows up in the exponents in eqs.(21, 23) we use the following two 'master formulae' (13]) for an expansion of exponentials 
of non-commuting operators:

$$
\begin{aligned}
e^{A+B}= & e^{A}+\int_{0}^{1} d \alpha e^{\alpha A} B e^{(1-\alpha) A} \\
& +\int_{0}^{1} d \alpha \int_{0}^{1-\alpha} d \beta e^{\alpha A} B e^{\beta A} B e^{(1-\alpha-\beta)} A+\ldots \\
{\left[B, e^{A}\right]=} & \int_{0}^{1} d \gamma e^{\gamma A}[B, A] e^{(1-\gamma) A} .
\end{aligned}
$$

Here B contains combinations of $D_{i}$ and A denotes the rest. Eqs. 21 23) as well as eq. (36) are independent of the color group. We found in 13 that for any $S U(N)$ the individual contributions from the ghosts and gluons to the order that we are interested in are

$$
\begin{gathered}
{\left[\log \operatorname{det}\left(-D^{2}\right)\right]_{\mathrm{E}}^{(2)}=-\int d^{3} x \sum^{M} e^{-s p^{2}}\left[I_{1}+I_{2}\right],} \\
{\left[\log (\operatorname{det} W)_{\mathrm{r}, \mathrm{n}}^{-1 / 2}\right]_{\mathrm{E}}^{(2)}=\int d^{3} x \sum^{M} e^{-s p^{2}}\left[2 I_{1}+2 I_{2}+\frac{I_{3}}{2}\right],}
\end{gathered}
$$

so that the 1-loop contribution to the kinetic energy in the electric sector is

$$
\left[S_{1-\mathrm{loop}}\right]_{\mathrm{E}}=\int d^{3} x \sum^{M} e^{-s p^{2}}\left[I_{1}+I_{2}+\frac{I_{3}}{2}\right] .
$$

The structures $I_{i}$ in eqs. (37) 38) are given by [13.

$$
\begin{aligned}
I_{1}= & s^{3} \int_{0}^{1} d \alpha\left\{-\frac{1}{2}+\alpha(1-\alpha)+\frac{2}{9} s p^{2}\left[1-\frac{3}{2} \alpha(1-\alpha)\right]\right\} \\
& \times \operatorname{Tr} e^{(1-\alpha) s \mathcal{A}^{2}}\left\{\mathcal{A}, E_{i}\right\} e^{\alpha s \mathcal{A}^{2}}\left\{\mathcal{A}, E_{i}\right\} \\
I_{2}= & -s^{2}\left(\frac{1}{2}-\frac{2}{9} s p^{2}\right) \operatorname{Tr} e^{s \mathcal{A}^{2}}\left(2 E_{i}^{2}+i\left\{\mathcal{A},\left[D_{i}, E_{i}\right]\right\}\right) \\
I_{3}= & 8 s^{2} \int_{0}^{1} d \alpha \frac{1}{2} \operatorname{Tr} e^{(1-\alpha) s \mathcal{A}^{2}} E_{i} e^{\alpha s \mathcal{A}^{2}} E_{i}
\end{aligned}
$$

where all matrices are in the adjoint representation. So far the gauge group has not been specified. For $S U(2)$ the result was presented in [13]. For $S U(3)$ we chose $A_{4}$ to be diagonal in the fundamental representation, eq. (26), and obtain the adjoint matrix $\mathcal{A}$ in eq. (31). The electric field is $E_{i}^{a b}=i f^{a c b} E_{i}^{c}$. The second term in the invariant $I_{2}$ is zero if the gluon fields obey the equation of motion. We leave it away for the time being but shall return to it later. After all integrations and the summation over all Matsubara frequencies, we find the following structure for the electric kinetic energy:

$$
\begin{aligned}
& {\left[S_{1-\text { loop }}\right]_{\mathrm{E}}=\int d^{3} x\left\{\left[\left(E_{i}^{1}\right)^{2}+\left(E_{i}^{2}\right)^{2}\right] \lambda_{1}(\nu)\right.} \\
& \quad+\left[\left(E_{i}^{4}\right)^{2}+\left(E_{i}^{5}\right)^{2}\right] \lambda_{4}(\nu)+\left[\left(E_{i}^{6}\right)^{2}+\left(E_{i}^{7}\right)^{2}\right] \lambda_{6}(\nu) \\
& \left.\quad+\left(E_{i}^{3}\right)^{2} \lambda_{3}(\nu)+\left(E_{i}^{8}\right)^{2} \lambda_{8}(\nu)+E_{i}^{3} E_{i}^{8} \lambda_{38}(\nu)\right\} .
\end{aligned}
$$

All functions $\lambda_{j}(\nu)$ are functions of the rescaled eigenvalues of $A_{4}^{\text {adj }}, \nu=\nu_{1}, \nu_{2}, \nu_{3}$, eq. (35). For the explicit sums over the Matsubara frequencies, one has to define the range of definition for the $\phi_{n}$, respectively the $\nu_{n}$. This defines the functional form of the $\lambda_{j}$. In this section we shall consider the range $0 \leq\left|\phi_{i}\right| \leq 2 \pi T$, which corresponds to $0 \leq\left|A_{4}^{3}\right|, \sqrt{3}\left|A_{4}^{8}\right| \leq 2 \pi T$,. Outside this interval the functions have to be computed separately. We would like to stress once more that we do not expect $\mathrm{Z}(3)$ symmetric results here, as we obtained them for the potential (34), which would be equivalent to invariance under large time-dependent gauge transformations. We broke this symmetry by making the background $A_{i}$ components static. As discussed before, to recover this symmetry one has to find the action to all orders in time derivatives.

We start by looking at the coefficients in front of the electric field orthogonal to $A_{4}, E_{i}^{1-2,4-7}$, namely $\lambda_{1,4,6}$. We shall split the results into a contribution from the non-zero Matsubara frequencies (denoted by a prime) and into the $\omega_{k=0}$ contribution as $\lambda_{i}=\lambda_{i}^{\prime}+\lambda_{i 0}$, where $i=1,4,6$. We find that as long as the individual eigenvalues $\nu_{i}$ are either between 0 and 1 or between -1 and 0 then the functional form of the $\lambda_{i}^{\prime}$ is given by

$$
\begin{aligned}
\lambda_{1}^{\prime}(\nu)= & -\frac{11}{96 \pi^{2} T}\left[6\left(\gamma_{\mathrm{E}}-\log \mu\right)+2 \psi\left(\frac{\nu_{1}}{2}\right)+2 \psi\left(-\frac{\nu_{1}}{2}\right)\right. \\
& \left.+\psi\left(\frac{\nu_{2}-\nu_{3}}{2}\right)+\psi\left(-\frac{\nu_{2}-\nu_{3}}{2}\right)\right], \\
\lambda_{4}^{\prime}(\nu)=- & \frac{11}{96 \pi^{2} T}\left[6\left(\gamma_{\mathrm{E}}-\log \mu\right)+2 \psi\left(\frac{\nu_{2}}{2}\right)+2 \psi\left(-\frac{\nu_{2}}{2}\right)\right. \\
& \left.+\psi\left(\frac{\nu_{1}+\nu_{3}}{2}\right)+\psi\left(-\frac{\nu_{1}+\nu_{3}}{2}\right)\right], \\
\lambda_{6}^{\prime}(\nu)=- & \frac{11}{96 \pi^{2} T}\left[6\left(\gamma_{\mathrm{E}}-\log \mu\right)+2 \psi\left(\frac{\nu_{3}}{2}\right)+2 \psi\left(-\frac{\nu_{3}}{2}\right)\right. \\
& \left.+\psi\left(\frac{\nu_{1}+\nu_{2}}{2}\right)+\psi\left(-\frac{\nu_{1}+\nu_{2}}{2}\right)\right] .
\end{aligned}
$$

Here $\psi$ is the digamma function

$$
\psi(\nu)=\frac{\partial}{\partial \nu} \log \Gamma(\nu)
$$

For the $\lambda_{i 0}$ their functional form depends on the region of definition of each of the three $\nu_{i}$ separately. It is, however, possible to express them in terms of the $\phi_{i}$ before specifying the intervals for the latter:

$$
\begin{aligned}
\lambda_{10}= & \frac{1}{2 \pi^{2}}\left[\frac{5 \pi}{3\left|\phi_{1}\right|}+\frac{\left|\phi_{2}\right| \pi\left(10 \phi_{2}^{3}+23 \phi_{2}^{2} \phi_{3}+10 \phi_{2} \phi_{3}^{2}+\phi_{3}^{3}\right)}{12 \phi_{2}\left(\phi_{2}-\phi_{3}\right)\left(\phi_{2}+\phi_{3}\right)^{3}}\right. \\
& \left.-\frac{\left|\phi_{3}\right| \pi\left(\phi_{2}^{3}+10 \phi_{2}^{2} \phi_{3}+23 \phi_{2} \phi_{3}^{2}+10 \phi_{3}^{3}\right)}{12 \phi_{3}\left(\phi_{2}-\phi_{3}\right)\left(\phi_{2}+\phi_{3}\right)^{3}}\right], \\
\lambda_{40}= & \frac{1}{2 \pi^{2}}\left[\frac{5 \pi}{3\left|\phi_{2}\right|}+\frac{\left|\phi_{3}\right| \pi\left(-10 \phi_{3}^{3}+23 \phi_{3}^{2} \phi_{1}-10 \phi_{3} \phi_{1}^{2}+\phi_{1}^{3}\right)}{12 \phi_{3}\left(\phi_{1}+\phi_{3}\right)\left(\phi_{1}-\phi_{3}\right)^{3}}\right. \\
& \left.+\frac{\left|\phi_{1}\right| \pi\left(-\phi_{3}^{3}+10 \phi_{3}^{2} \phi_{1}-23 \phi_{3} \phi_{1}^{2}+10 \phi_{1}^{3}\right)}{12 \phi_{1}\left(\phi_{1}+\phi_{3}\right)\left(\phi_{1}-\phi_{3}\right)^{3}}\right],
\end{aligned}
$$




$$
\begin{aligned}
\lambda_{60}= & \frac{1}{2 \pi^{2}}\left[\frac{5 \pi}{3\left|\phi_{3}\right|}+\frac{\left|\phi_{1}\right| \pi\left(10 \phi_{1}^{3}-23 \phi_{1}^{2} \phi_{2}+10 \phi_{1} \phi_{2}^{2}-\phi_{2}^{3}\right)}{12 \phi_{1}\left(\phi_{1}+\phi_{2}\right)\left(\phi_{1}-\phi_{2}\right)^{3}}\right. \\
& \left.+\frac{\left|\phi_{2}\right| \pi\left(\phi_{1}^{3}-10 \phi_{1}^{2} \phi_{2}+23 \phi_{1} \phi_{2}^{2}-10 \phi_{2}^{3}\right)}{12 \phi_{2}\left(\phi_{1}+\phi_{2}\right)\left(\phi_{1}-\phi_{2}\right)^{3}}\right]
\end{aligned}
$$

Note that there is no explicit factor $1 / \mathrm{T}$, since the variables are not rescaled yet. One can easily see that these functions can be obtained from each other as $\lambda_{40}=$ $\lambda_{10}\left(\phi_{1} \rightarrow \phi_{2}, \phi_{2} \rightarrow-\phi_{1}\right)$ and $\lambda_{60}=\lambda_{40}\left(\phi_{2} \leftrightarrow \phi_{3}\right)$. To show how the functions (47.49) depend on the region of definition of $\phi_{i}$ we show some examples. If all three $\nu_{i}=\phi_{i} /(2 \pi T)$ are defined to be in the interval from 0 to +1 , then we have

$$
\begin{aligned}
& \lambda_{10}=\frac{1}{12 \pi^{2} T}\left[\frac{5}{\nu_{1}}-\frac{\nu_{2}^{2}}{\left(\nu_{2}+\nu_{3}\right)^{3}}+\frac{\nu_{2}}{\left(\nu_{2}+\nu_{3}\right)^{2}}+\frac{9}{4\left(\nu_{2}+\nu_{3}\right)}\right], \\
& \lambda_{40}=\frac{1}{12 \pi^{2} T}\left[\frac{5}{\nu_{2}}+\frac{11}{4\left(\nu_{1}+\nu_{3}\right)}\right] \\
& \lambda_{60}=\frac{1}{12 \pi^{2} T}\left[\frac{5}{\nu_{3}}+\frac{11}{4\left(\nu_{1}+\nu_{2}\right)}\right] .
\end{aligned}
$$

If all three $\nu_{i}$ lie between -1 and 0 , then the above functions just change their global sign. If we put $\nu_{1}$ and $\nu_{3}$ in the positive region from 0 to 1 and $\nu_{2}$ into the negative one from -1 to 0 , then the functions change to

$$
\begin{aligned}
& \lambda_{10}=\frac{1}{12 \pi^{2} T}\left[\frac{5}{\nu_{1}}+\frac{11}{4\left(\nu_{3}-\nu_{2}\right)}\right], \\
& \lambda_{40}=\frac{1}{12 \pi^{2} T}\left[-\frac{5}{\nu_{2}}+\frac{11}{4\left(\nu_{1}+\nu_{3}\right)}\right], \\
& \lambda_{60}=\frac{1}{12 \pi^{2} T}\left[\frac{5}{\nu_{3}}-\frac{\nu_{1}^{2}}{\left(\nu_{1}-\nu_{2}\right)^{3}}+\frac{\nu_{1}}{\left(\nu_{1}-\nu_{2}\right)^{2}}+\frac{9}{4\left(\nu_{1}-\nu_{2}\right)}\right] .
\end{aligned}
$$

Adding up the contributions from the non-zero Matsubara frequencies, eqs. (43.45), and the $\lambda_{i 0}$ terms from eqs. (4749), one finds that the functions are not symmetric in the $\nu_{i}$ between 0 and 1 (or between -1 and 0 ), which would have been the requirement for the $\mathrm{Z}(3)$ equivalence.

The remaining coefficient functions $\lambda_{3,8,38}(\nu)$ in eq. (42), which are connected to the electric field parallel to $A_{4}$, viz. $E_{i}^{3}$ and $E_{i}^{8}$, can be expressed in terms of the function

$$
h(\nu)=\frac{1}{|\nu|}+2\left(\log \mu-\gamma_{\mathrm{E}}\right)-\psi(\nu)-\psi(-\nu) .
$$

In the positive region $0 \leq \nu \leq 1$ we can use that $\psi(\nu)+$ $1 / \nu=\psi(1+\nu)$ and eq. (52) becomes

$$
\begin{aligned}
h(\nu) & =2\left(\log \mu-\gamma_{\mathrm{E}}\right)-\psi(\nu)-\psi(1-\nu) \\
& \equiv 2 \log \mu+H(\nu) \\
H(\nu) & =-\psi(\nu)-\psi(1-\nu)-2 \gamma_{\mathrm{E}} .
\end{aligned}
$$

Inside the interval $0 \leq \nu \leq 1$ the function is symmetric with respect to the replacement $\nu \rightarrow 1-\nu$. Outside

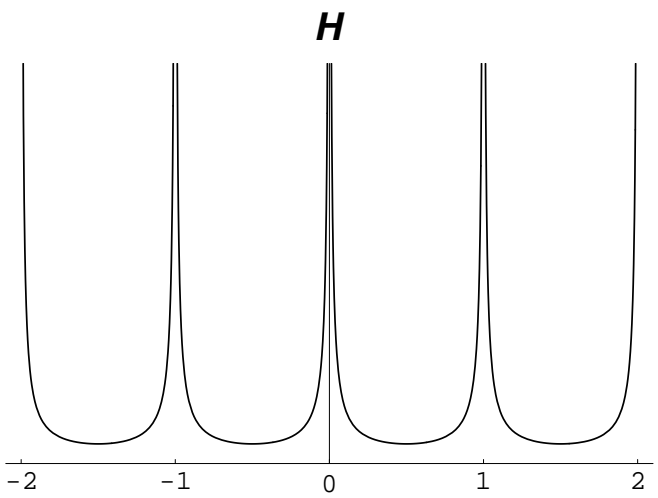

FIG. 2: The periodic function $H(\nu)$ plotted versus $\nu$.

this interval the function is continued by periodicity with period 1. We plot the cutoff-independent function $H(\nu)$ in Fig. 2. We would like to stress that the term $1 / \nu$ is always the (sole) contribution of the zero Matsubara frequency $\omega_{0}$.

If we keep all the $\nu_{n}$ either between -1 and 0 or between 0 and 1 we find the following functional form for the coefficients in the parallel sector:

$$
\begin{aligned}
\lambda_{3}(\nu) & =\frac{11}{192 \pi^{2} T}\left[4 h\left(\nu_{1}\right)+h\left(\nu_{2}\right)+h\left(\nu_{3}\right)\right], \\
\lambda_{8}(\nu) & =\frac{11}{64 \pi^{2} T}\left[h\left(\nu_{2}\right)+h\left(\nu_{3}\right)\right] \\
\lambda_{38}(\nu) & =\frac{11}{32 \sqrt{3} \pi^{2} T}\left[h\left(\nu_{2}\right)-h\left(\nu_{3}\right)\right] .
\end{aligned}
$$

They are periodic functions in the eigenvalues of $A_{4}^{\text {adj }}$.

Similar to the case of $S U(2)$ discussed in 13], the periodicity in the parallel sector comes from the fact that the electric field components $E_{i}^{3,8}=\partial_{i} A_{4}^{3,8}+$ $f^{(3,8) c(3,8)} A_{i}^{c} A_{4}^{3,8}=\partial_{i} A_{4}^{3,8}$ do not dependent on $A_{i}$ and are by themselves invariant under special time-dependent gauge transformation (8) leading to the shift of $A_{4}^{3,8}$ by integers. Since the action has to be invariant under (8), it can only happen if the coefficient functions $\lambda_{3,8,38}(\nu)$ are periodic functions of $\nu_{m}$ which indeed they are owing to the periodicity of the functions $h(\nu)$. The rest electric field components necessarily contain the $A_{i}$ fields which become fast time-dependent under the transformation (8), see eqs. (1116). The full electric field is $E_{i}^{a}=D_{i}^{a b} A_{4}^{b}-\dot{A}_{i}^{a}$. Therefore, the transformation (8) changes the components $E_{i}^{1,2,4,5,6,7}$, and the invariance under (8) does not request that the corresponding coefficient functions $\lambda_{1,4,6}(\nu)$ are periodic. The $Z(3)$ symmetry in the 'transverse' electric sector has to take place only when one collects all powers of $\dot{A}_{i}$.

All functions $\lambda_{1}, \ldots, \lambda_{8}$ (but not the cross-term $\lambda_{38}$ ) contain the same UV divergent contribution

$$
\frac{11}{16 \pi^{2} T} \log \mu \text {. }
$$


The parameter $\mu$ is the UV-cutoff in divergent series:

$$
\sum_{k=1}^{\infty} \frac{1}{k} \rightarrow \sum_{k=1}^{\mu} \frac{1}{k} \equiv \log \mu
$$

and is related to the Pauli-Villars mass as

$$
\mu=\frac{M}{4 \pi T} e^{\gamma_{\mathrm{E}}}
$$

This subtraction scale for the running coupling constant has been known previously [19] and was also obtained in 13.

The divergence (58) is necessary to cancel the UV divergence from the running coupling constant in the tree level action:

$$
\begin{aligned}
-\frac{F_{\mu \nu}^{2}}{4 g^{2}(M)} & =-\frac{F_{\mu \nu}^{2}}{8 \pi^{2}} \log \frac{M}{\Lambda} \frac{11}{12} N_{c} \\
& =-\left(E_{i}^{2}+B_{i}^{2}\right) \frac{11}{16 \pi^{2}} \log \frac{M}{\Lambda} .
\end{aligned}
$$

If we add the tree-level and the 1-loop action then the result should be UV finite. This is obtained by choosing the scale $M$ in eq. (61) to be equal to the Pauli-Villars mass, which corresponds to the evaluation of the running coupling constant at the scale $4 \pi T / \exp \left(\gamma_{\mathrm{E}}\right)$. In the effective action we then have to replace the Pauli-Villars cutoff $M$ by $\Lambda$ in all our functions $\lambda_{i}$ from eq. (42).

\section{GAUGE INVARIANT STRUCTURES}

Written in the form above the 1-loop action eq. (42) does not look covariant. This is, however, a consequence of the fact that we chose $A_{4}$ to be diagonal. It is easy to show that eq. (42) can be rewritten as

$$
\left[S_{1-\text { loop }}\right]_{\mathrm{E}}=\int d^{3} x \sum_{i=1}^{5} \Phi_{i} f_{i}\left(\nu_{1}, \nu_{2}, \nu_{3}\right)
$$

with the following five invariant structures:

$$
\begin{aligned}
& \Phi_{1}=\operatorname{Tr}\left[E_{i} A_{4}\right] \operatorname{Tr}\left[E_{i} A_{4}\right] \\
& \Phi_{2}=\operatorname{Tr}\left[E_{i} E_{i}\right] \\
& \Phi_{3}=\operatorname{Tr}\left[E_{i} A_{4} E_{i} A_{4}\right] \\
& \Phi_{4}=\operatorname{Tr}\left[E_{i} A_{4}{ }^{2} E_{i} A_{4}{ }^{2}\right] \\
& \Phi_{5}=\operatorname{Tr}\left[E_{i} A_{4}{ }^{3}\right] \operatorname{Tr}\left[E_{i} A_{4}{ }^{3}\right]
\end{aligned}
$$

All the traces are over matrices in the adjoint representation. For our particular choice (26) for $A_{4}$ these struc- tures are composed as follows:

$$
\begin{aligned}
& \Phi_{1}=4 \pi^{2} T^{2}\left[\sqrt{3} E_{i}^{8}\left(\nu_{2}-\nu_{3}\right)+E_{i}^{3}\left(2 \nu_{1}+\nu_{2}+\nu_{3}\right)\right]^{2}, \\
& \Phi_{2}=3\left[\left(E_{i}^{a} E_{i}^{a}\right)\right], \\
& \Phi_{3}=2 \pi^{2} T^{2}\left[-2 \nu_{2} \nu_{3}\left(E_{i}^{1^{2}}+E_{i}^{2^{2}}\right)+2 \nu_{1} \nu_{3}\left(E_{i}^{4^{2}}+E_{i}^{5^{2}}\right)\right. \\
& +2 \nu_{1} \nu_{2}\left(E_{i}^{6^{2}}+E_{i}^{7^{2}}\right)+E_{i}^{3^{2}}\left(4 \nu_{1}^{2}+\nu_{2}^{2}+\nu_{3}^{2}\right) \\
& \left.+3 E_{i}^{8^{2}}\left(\nu_{2}^{2}+\nu_{3}^{2}\right)+2 \sqrt{3} E_{i}^{3} E_{i}^{8}\left(\nu_{2}-\nu_{3}\right)\right], \\
& \Phi_{4}=8 \pi^{4} T^{4}\left[2 \nu_{2}{ }^{2} \nu_{3}{ }^{2}\left(E_{i}^{1^{2}}+E_{i}^{2^{2}}\right)+2 \nu_{1}^{2} \nu_{3}{ }^{2}\left(E_{i}^{4^{2}}+E_{i}^{5^{2}}\right)\right. \\
& +2 \nu_{1}{ }^{2} \nu_{2}{ }^{2}\left(E_{i}^{6^{2}}+E_{i}^{7^{2}}\right)+E_{i}^{3^{2}}\left(4 \nu_{1}{ }^{4}+\nu_{2}{ }^{4}+\nu_{3}{ }^{4}\right) \\
& \left.+3 E_{i}^{8^{2}}\left(\nu_{2}{ }^{4}+\nu_{3}{ }^{4}\right)+2 \sqrt{3} E_{i}^{3} E_{i}^{8}\left(\nu_{2}{ }^{4}-\nu_{3}{ }^{4}\right)\right], \\
& \Phi_{5}=64 \pi^{6} T^{6}\left[\sqrt{3} E_{i}^{8}\left(\nu_{2}{ }^{3}-\nu_{3}{ }^{3}\right)+E_{i}^{3}\left(2 \nu_{1}{ }^{3}+\nu_{2}{ }^{3}+\nu_{3}{ }^{3}\right)\right]^{2} \text {. }
\end{aligned}
$$

Since the coefficients $f_{i}$ in front of these structures in eq. (62), which reproduce eq. (42), are rather lengthy we do not write them down explicitly here. But it is easy to reconstruct them with eq. (64). We would like to stress, however, that the UV logarithm is now only contained in the coefficient $f_{2}$ in front of $\Phi_{2}$. This is expected, since only that structure shows up in the classical action eq. (61).

\section{THE MAGNETIC SECTOR}

To get the magnetic fields in the effective action we have to expand eqs. (21) 23) to the quartic order in $D_{i}$. The basic idea of the calculation is, again, to use master equations (36) to drag covariant derivatives $D_{i}$ to the right. What enters in the expansion is

$$
\begin{aligned}
D_{i} e^{s \mathcal{A}^{2}} & =e^{s \mathcal{A}^{2}} D_{i}-i s \int_{0}^{1} d \delta e^{\delta s \mathcal{A}^{2}}\left\{\mathcal{A}, E_{i}\right\} e^{(1-\delta) s \mathcal{A}^{2}}, \\
D_{i} D_{j} e^{s \mathcal{A}^{2}} & =e^{s \mathcal{A}^{2}} D_{i} D_{j}-i s \int_{0}^{1} d \delta e^{\delta s \mathcal{A}^{2}}\left[D_{i} D_{j}, \mathcal{A}^{2}\right] e^{(1-\delta) s \mathcal{A}^{2}}
\end{aligned}
$$

where

$$
\left[D_{i} D_{j}, \mathcal{A}^{2}\right]=-i D_{i}\left\{\mathcal{A}, E_{j}\right\}-i\left\{\mathcal{A}, E_{i}\right\} D_{j} .
$$

In this way one ultimately obtains gauge-invariant combinations of the electric field in the fourth power, mixed terms containing both electric and magnetic fields, derivatives of the electric field and, finally, magnetic field squared. In this paper we restrict ourselves to the latter terms quadratic in the magnetic field $B_{i}$. This means that we treat $D_{i}$ and $A_{4}$ as commuting operators and neglect the commutators $\left[D_{i}, A_{4}\right]$. Moreover, unlike for the electric field, we would like to restrict ourselves to a magnetic field parallel to $A_{4}$, i.e. $B_{i}=B_{i \|}$. In that case we see from the Bianchi identity $\left[F_{i j}, A_{4}\right]=$ $i\left(\left[D_{i}, E_{j}\right]-\left[D_{j}, E_{i}\right]\right)$ that we can neglect derivatives of 
the electric field as well. Hence we can also treat $D_{i}$ and $F_{i j}$ as commuting operators.

As shown in 13. there is only one structure that contributes to quadratic order in the magnetic field:

$$
\begin{gathered}
{\left[S_{1-\text { loop }}^{(2)}\right]_{\mathrm{M}}=\left[\log (\operatorname{det} W)_{\mathrm{r}, \mathrm{n}}^{-1 / 2}+\log \operatorname{det}\left(-D^{2}\right)_{\mathrm{r}, \mathrm{n}}\right]_{\mathrm{M}}^{(2)}(66)} \\
=\frac{11}{48 \pi^{3 / 2}} \int d^{3} x \sum_{k=-\infty}^{\infty} \int_{0}^{\infty} \frac{d s}{\sqrt{s}} \operatorname{Tr}\left(1-e^{-s M^{2}}\right)\left(e^{s \mathcal{A}^{2}} B_{k} B_{k}\right) .
\end{gathered}
$$

Eq. (66) is so far independent of the gauge group. For $S U(3)$ we have the matrix $\mathcal{A}$ given by (31), and the magnetic field is in the adjoint representation, $B_{i}^{a b}=i f^{a c b} B_{i}^{c}$ where $B_{i}^{c}$ is as in eq. (24). After the integrations over $s$ and $p$ and the summation over the Matsubara frequencies $\omega_{k}$ we find the following result:

$$
\begin{gathered}
{\left[S_{1-\text { loop }}\right]_{\mathrm{M}}=\int d^{3} x\left\{\left(B_{i}^{3}\right)^{2} \kappa_{3}(\nu)\right.} \\
\left.+\left(B_{i}^{8}\right)^{2} \kappa_{8}(\nu)+B_{i}^{3} B_{i}^{8} \kappa_{38}(\nu)\right\} .
\end{gathered}
$$

Again the coefficients $\kappa_{i}$ are functions of the rescaled eigenvalues of $A_{4}^{\text {adj }}, \nu=\nu_{1}, \nu_{2}, \nu_{3}$, and their functional form will depend on the region of definition of them. As before we chose $0 \leq\left|\phi_{i}\right| \leq 2 \pi T$. The fact that the result (67) looks non-invariant is again a consequence of the diagonal form of $A_{4}$. However, eq. (67) can be rewritten in terms of the invariants (63) with an obvious replacement $E_{i}^{3,8} \rightarrow B_{i}^{3,8}$ and $E_{i}^{1,4,6} \rightarrow 0$. All the functions $\kappa_{j}$ are linear combinations of the function $h$, defined in eq. (52), with arguments $\nu_{1,2,3}$ :

$$
\begin{aligned}
\kappa_{3}(\nu) & =\frac{11}{192 \pi^{2} T}\left[4 h\left(\nu_{1}\right)+h\left(\nu_{2}\right)+h\left(\nu_{3}\right)\right]=\lambda_{3}(\nu), \\
\kappa_{8}(\nu) & =\frac{33}{192 \pi^{2} T}\left[h\left(\nu_{2}\right)+h\left(\nu_{3}\right)\right]=\lambda_{8}(\nu), \\
\kappa_{38}(\nu) & =\frac{11}{32 \sqrt{3} \pi^{2} T}\left[h\left(\nu_{2}\right)-h\left(\nu_{3}\right)\right]=\lambda_{38}(\nu) .
\end{aligned}
$$

All of these functions are symmetric in the rescaled eigenvalues $\nu$ of $A_{4}^{\text {adj }}$, which corresponds to the equivalence of the $\mathrm{Z}(3)$-symmetric points. The reason for this is similar to the one we discussed for the parallel electric sector. Since the magnetic field

$$
B_{i}^{a}=\frac{1}{2} \epsilon_{i j k}\left(\partial_{j} A_{k}^{a}-\partial_{k} A_{j}^{a}+\epsilon^{a b c} A_{j}^{b} A_{k}^{c}\right)
$$

does not have any explicit time dependence, a fast timedependent gauge transformation does not affect $B_{i}{ }^{2}$, neither for static nor for time dependent $A_{i}$ fields. The invariance of the action with respect to the special timedependent gauge transformation (8) requires then that eq. (67) is $Z(3)$-symmetric, which amounts to the periodicity of all functions $\kappa(\nu)$. Indeed, they are all periodic.

The functions $\kappa_{3}, \kappa_{8}$ have the same UV divergent contribution

$$
\frac{11}{16 \pi^{2} T} \log \mu
$$

while the cross-term $\kappa_{38}$ is UV finite. This divergence removes the one from the tree level action, eq. (61). Adding eq. (61) and eq. (67) corresponds to the evaluation of the running coupling constant at the scale $4 \pi T / \exp \left(\gamma_{\mathrm{E}}\right)$. In the effective action we then have to replace the PauliVillars cutoff $M$ by $\Lambda$ in all our functions $\kappa_{i}$ from eq. (67).

\section{INVARIANCE UNDER PERMUTATIONS OF COLOR AXES}

An important check of the results (4267) is their invariance under color rotations corresponding to the permutation of color axes. We can for example rotate from the color- 1 axes to the color- 4 axes, or from the color- 4 axes to the color- 6 axes. This corresponds to finding the matrices $S$ and $U$ such that:

$$
\begin{aligned}
S^{\dagger} \lambda^{1} S & =\lambda^{4}, \\
U^{\dagger} \lambda^{4} U & =\lambda^{6} .
\end{aligned}
$$

They are given by

$$
S=\left(\begin{array}{ccc}
1 & 0 & 0 \\
0 & 0 & 1 \\
0 & -1 & 0
\end{array}\right) \quad, \quad U=\left(\begin{array}{ccc}
0 & 1 & 0 \\
-1 & 0 & 0 \\
0 & 0 & 1
\end{array}\right)
$$

We shall show explicitly how this works for the first of these two rotations, namely the one with $S$ around $\lambda^{1}$. We chose $A_{4}$ to be diagonal in the fundamental representation, eq. (26),

$$
A_{4}=A_{4}^{3} t^{3}+A_{4}^{8} t^{8} .
$$

Under the rotation $S^{\dagger} A_{4} S$ we find the transformations

$$
A_{4}^{3} \rightarrow \frac{A_{4}^{3}+\sqrt{3} A_{4}^{8}}{2} \equiv \tilde{A}_{4}^{3}, A_{4}^{8} \rightarrow \frac{3 A_{4}^{3}-\sqrt{3} A_{4}^{8}}{2 \sqrt{3}} \equiv \tilde{A}_{4}^{8} \cdot(75)
$$

The electric and magnetic fields transform with $\mathcal{S}^{a b}=$ $\frac{1}{2} \operatorname{Tr}\left(S^{\dagger} \lambda^{a} S \lambda^{b}\right)$ to

$$
\begin{gathered}
\mathcal{S}^{a b} E_{i}^{b} \equiv \tilde{E}_{i}^{a} \\
=\left(E_{i}^{4}, E_{i}^{5}, \frac{E_{i}^{3}+\sqrt{3} E_{i}^{8}}{2},-E_{i}^{1},-E_{i}^{2},-E_{i}^{6}, E_{i}^{7}, \frac{\sqrt{3} E_{i}^{3}-E_{i}^{8}}{2}\right) \\
\mathcal{S}^{a b} B_{i}^{b} \equiv \tilde{B}_{i}^{a} \\
=\left(0,0, \frac{B_{i}^{3}+\sqrt{3} B_{i}^{8}}{2}, 0,0,0,0, \frac{\sqrt{3} B_{i}^{3}-B_{i}^{8}}{2}\right)
\end{gathered}
$$

We hence see that the structures orthogonal and parallel to $A_{4}$ transform separately. We shall start by checking the results in the electric sector. For the orthogonal part we have to show that

$$
\begin{aligned}
& \lambda_{1}\left(A_{4}^{3}, A_{4}^{8}\right)=\lambda_{4}\left(\tilde{A}_{4}^{3}, \tilde{A}_{4}^{8}\right), \\
& \lambda_{4}\left(A_{4}^{3}, A_{4}^{8}\right)=\lambda_{1}\left(\tilde{A}_{4}^{3}, \tilde{A}_{4}^{8}\right), \\
& \lambda_{6}\left(A_{4}^{3}, A_{4}^{8}\right)=\lambda_{6}\left(\tilde{A}_{4}^{3}, \tilde{A}_{4}^{8}\right),
\end{aligned}
$$


and for the parallel part the requirement is

$$
\begin{aligned}
& E_{i}^{3^{2}} \lambda_{3}\left(A_{4}^{3}, A_{4}^{8}\right)+E_{i}^{8^{2}} \lambda_{8}\left(A_{4}^{3}, A_{4}^{8}\right)+E_{i}^{3} E_{i}^{8} \lambda_{38}\left(A_{4}^{3}, A_{4}^{8}\right) \\
& =\tilde{E}_{i}^{3} \lambda_{3}\left(\tilde{A}_{4}^{3}, \tilde{A}_{4}^{8}\right)+\tilde{E}_{i}^{8} \lambda_{8}\left(\tilde{A}_{4}^{3}, \tilde{A}_{4}^{8}\right) \\
& \quad+\tilde{E}_{i}^{3} \tilde{E}_{i}^{8} \lambda_{38}\left(\tilde{A}_{4}^{3}, \tilde{A}_{4}^{8}\right) .
\end{aligned}
$$

There is one additional subtlety, however. Here we expressed the functions through $A_{4}^{3}, A_{4}^{8}$, instead of through the eigenvalues $\nu_{i}$. We mentioned before that the functional form of the $\lambda_{i}$ depends on the interval of definition for the individual $\nu_{i}$. So if we compare functions of $A_{4}^{3}, A_{4}^{8}$ to functions of $\tilde{A}_{4}^{3}, \tilde{A}_{4}^{8}$, we have to classify these functions according to their support in $\nu_{i}$, i.e. the $\nu_{i}$ 's that correspond to $A_{4}^{3}, A_{4}^{8}$ can lie in different intervals than the $\nu_{i}$ 's corresponding to $\tilde{A}_{4}^{3}, \tilde{A}_{4}^{8}$. For a comparison we then have to choose different functional forms of the $\lambda_{i}$.

It turns out, however, that as long as $A_{4}^{3} /(2 \pi T)$ and $\sqrt{3} A_{4}^{8} /(2 \pi T)$ are between -1 and 1 (then also the $\nu_{i}$ are in that region) we can check the contribution of the zero Matsubara mode and of the non-zero modes separately.

We start by checking the contribution of the zero Matsubara mode in the orthogonal electric sector, eqs.47. 491). It is independent of the region of definition of $A_{4}^{3} /(2 \pi T)$ and $A_{4}^{8} /(2 \pi T)$, since it is not rescaled. First we express it in terms of $A_{4}^{3}$ and $A_{4}^{8}$, then we make the rotation to $\tilde{A}_{4}^{3}, \tilde{A}_{4}^{8}$ according to eq. (75). And we find indeed that $\lambda_{10}\left(A_{4}^{3}, A_{4}^{8}\right)=\lambda_{40}\left(\tilde{A}_{4}^{3}, \tilde{A}_{4}^{8}\right), \lambda_{40}\left(A_{4}^{3}, A_{4}^{8}\right)=$ $\lambda_{10}\left(\tilde{A}_{4}^{3}, \tilde{A}_{4}^{8}\right)$ and $\lambda_{60}\left(A_{4}^{3}, A_{4}^{8}\right)=\lambda_{60}\left(\tilde{A}_{4}^{3}, \tilde{A}_{4}^{8}\right)$. The $\lambda_{i}^{\prime}$ in eqs. 43 45) have the same functional form for individual $\nu_{i}$ in the positive or negative region. Therefore, we first express them in terms of $A_{4}^{3} /(2 \pi T)$ and $A_{4}^{8} /(2 \pi T)$ and then make the rotation eq. (75). As expected we find that $\lambda_{1}^{\prime}\left(A_{4}^{3}, A_{4}^{8}\right)=\lambda_{4}^{\prime}\left(\tilde{A}_{4}^{3}, \tilde{A}_{4}^{8}\right), \lambda_{4}^{\prime}\left(A_{4}^{3}, A_{4}^{8}\right)=\lambda_{1}^{\prime}\left(\tilde{A}_{4}^{3}, \tilde{A}_{4}^{8}\right)$ and $\lambda_{6}^{\prime}\left(A_{4}^{3}, A_{4}^{8}\right)=\lambda_{6}^{\prime}\left(\tilde{A}_{4}^{3}, \tilde{A}_{4}^{8}\right)$. Therefore, the electric sector orthogonal to $A_{4}$ is indeed invariant under color rotations.

The functional form of eqs.(55 57) for the electric field parallel to $A_{4}$ depends on the region of definition of each of the three $\nu_{n}$. It is again the contribution of the zero Matsubara frequency which is responsible for that. It comes in as the $1 /|\nu|$ term in the definition of the function $h(\nu)$ in eq. (52). If $0 \leq \nu \leq 1$ then one has $+1 / \nu$, while it becomes $-1 / \nu$ if $-1 \leq \nu \leq 0$. As discussed above, however, the color rotation eq. (75) can rotate individual $\nu_{n}$ from a positive region of definition into the negative one, and vice versa. This problem can be circumvented, however, by replacing the argument of the function $h\left(\nu_{n}\right)$ in the coefficients $\lambda_{3,8,38}(\nu)$ of eqs. (55.57) by the fractional part of $\nu_{n}$. Then it is easy to check that the invariance requirement eq. (79) is fulfilled for each value of $A_{4}^{3}$ and $\sqrt{3} A_{4}^{8}$ between $-2 \pi T$ and $2 \pi T$, where the functions $h\left(\nu_{n}\right)$ are defined.

What remains is to check the results for the magnetic sector of the theory, eqs. 67 [68). Since the invariance requirements eqs. (78) remain the same in the electric sector if we replace $\lambda_{i} \rightarrow \kappa_{i}$ and $E_{i} \rightarrow B_{i}$ we have already shown the invariance of the magnetic sector parallel to $A_{4}$, since according to eq. (68) $\kappa_{3,8,38}=\lambda_{3,8,38}$.

Similarly one checks the invariance under other color rotations, for example the one with $U$ in (73).

\section{THE "EQUATION OF MOTION" TERM}

We finally return to the second term in the invariant $I_{2}$, eq. (40). In the derivation of our results in the previous sections we ignored this term since it vanishes if the gluon fields obey the equation of motion. Its contribution to the effective action is

$$
\begin{aligned}
& S_{\mathrm{EoM}}=\int d^{3} x \sum_{k=-\infty}^{\infty} \int \frac{d^{3} p}{(2 \pi)^{3}} \int_{0}^{\infty} \frac{d s}{s} e^{-s p^{2}} \\
& \times\left[-s^{2}\left(\frac{1}{2}-\frac{2}{9} s p^{2}\right) \operatorname{Tr} e^{s \mathcal{A}^{2}}\left(i\left\{\mathcal{A},\left[D_{i}, E_{i}\right]\right\}\right)\right],
\end{aligned}
$$

which after integrations and summation yields

$$
S_{\mathrm{EoM}}=\int d^{3} x \frac{1}{8 \pi}\left[\frac{\left(D_{i} E_{i}\right)^{a} A_{4}^{a}}{\pi T}-\frac{\left(D_{i} E_{i}\right)^{b} A_{4}^{b}}{\sqrt{A_{4}^{c} A_{4}^{c}}}\right]
$$

Here the first term comes exclusively from the nonzero Matsubara frequencies, while the second term is the contribution of the zero Matsubara frequency alone. The result is similar to the one that we obtained for the case of $S U(2)$. The only difference is the prefactor: while it is $1 / 8$ here, it was $1 / 12$ in [13]. Equation (81) is zero if the classical equation of motion is satisfied. If the background field does not satisfy the equation of motion one can integrate eq. (81) by parts which yields:

$$
\begin{gathered}
S_{\mathrm{EoM}}=\frac{1}{8 \pi^{2}} \int d^{3} x\left\{E_{i}^{a} E_{i}^{a}\left(\frac{\pi}{\left|A_{4}\right|}-\frac{1}{T}\right)\right. \\
\left.+\frac{\pi}{\left|A_{4}\right|^{3}}\left(E_{i}^{a} A_{4}^{a}\right)\left(E_{i}^{b} A_{4}^{b}\right)-\partial_{i}\left(E_{i}^{a} A_{4}^{a}\left(\frac{\pi}{\left|A_{4}\right|}-\frac{1}{T}\right)\right)\right\}
\end{gathered}
$$

The last term here is a full derivative, and the remaining two have to be added to our results found before in the electric sector.

\section{COMPARISON WITH PREVIOUS WORK}

In a related work by Chapman [20] an effective action for the static modes in a pure Yang-Mills theory was obtained by means of a covariant derivative expansion. While we kept all orders of $A_{4}$ in the present work the author of 20] expands to quadratic order in the $A_{4}$ fields. For a comparison we hence have to expand our functions to quadratic order in $A_{4}$. The result for the electric sector 
is

$$
\begin{aligned}
& \frac{11 \zeta(3)}{768 \pi^{2} T}\left\{2 \frac{A_{4}^{3} A_{4}^{8}}{(2 \pi T)^{2}}\left[-E_{i}^{4^{2}}-E_{i}^{5^{2}}+E_{i}^{6^{2}}+E_{i}^{7^{2}}+8 \sqrt{3} E_{i}^{3} E_{i}^{8}\right]\right. \\
& +\frac{\left(A_{4}^{3}\right)^{2}}{(2 \pi T)^{2}}\left[8\left({E_{i}^{1}}^{2}+{E_{i}^{2}}^{2}\right)+36{E_{i}^{3}}^{2}+12 E_{i}^{8^{2}}\right. \\
& \left.+11\left(E_{i}^{4^{2}}+E_{i}^{5^{2}}+E_{i}^{6^{2}}+E_{i}^{7^{2}}\right)\right] \\
& +\frac{\left(A_{4}^{8}\right)^{2}}{(2 \pi T)^{2}}\left[4\left(E_{i}^{1^{2}}+E_{i}^{2^{2}}+E_{i}^{3^{2}}\right)+12 E_{i}^{8^{2}}\right. \\
& \left.\left.+3\left(E_{i}^{4^{2}}+E_{i}^{5^{2}}+E_{i}^{6^{2}}+E_{i}^{7^{2}}\right)\right]\right\}
\end{aligned}
$$

while in the magnetic sector we find

$$
\begin{aligned}
& \frac{11 \zeta(3)}{384 \pi^{2} T}\left\{8 \sqrt{3} \frac{A_{4}^{3} A_{4}^{8}}{(2 \pi T)^{2}} B_{i}^{3} B_{i}^{8}\right. \\
& \left.+\frac{\left(A_{4}^{3}\right)^{2}}{(2 \pi T)^{2}}\left[18 B_{i}^{3^{2}}+6 B_{i}^{8^{2}}\right]+\frac{\left(A_{4}^{8}\right)^{2}}{(2 \pi T)^{2}}\left[2 B_{i}^{3^{2}}+6 B_{i}^{8^{2}}\right]\right\} .
\end{aligned}
$$

This agrees with the results found in [20] if the gauge group is chosen to be $S U(3)$ and the magnetic field is restricted to be parallel to $A_{4}$.

While this paper was in preparation, ref. 21] appeared on the web, where a similar expansion in covariant derivatives of the effective action at high temperatures was performed, see also 22]. Many of our results coincide with theirs: in the electric sector the terms in the effective action where the electric field is parallel to the $A_{4}$ field, i.e. our functions $\lambda_{3,8,38}$, and our results in the (parallel) magnetic sector, $\kappa_{3,8,38}$. While we consider a parallel magnetic field, the authors of 21] have a general result. The corresponding functions in ref. 21] are periodic in $A_{4}^{3,8}$, but get an infinite contribution from the zero Matsubara frequencies.

\section{EFFECTIVE ACTION FOR THE EIGENVALUES OF THE POLYAKOV LINE}

\section{A. $\mathrm{SU}(3)$ case}

In the previous sections, we have computed the 1loop effective action for the Polyakov line $L(x)=$ $\exp \left(i A_{4}(x) / T\right)$ as a slowly varying element of $S U(3)$. Since $L(x)$ rotates under spatial gauge transformations (6) one has to introduce a nonzero $A_{i}$ background field in order to maintain gauge invariance of the effective action under spatial gauge transformations, and expand it in covariant derivatives $D_{i}$. If, however, only timeindependent $A_{i}$ 's are introduced, as it is required by static gauge invariance, the $Z(3)$ symmetry of the effective action is generally lost, as anticipated in section II and checked in section V. In order to restore the $Z(3)$ symmetry of the effective action, one needs to sum up all time derivatives of $A_{i}$, since the $Z(3)$ symmetry is a consequence of the invariance under fast time-dependent gauge transformations (8). This seems to be a formidable task which lies beyond the scope of this study; therefore we have to restrict ourselves to purely static $A_{4}, A_{i}$ fields at the cost that the $Z(3)$ symmetry of our effective action is not manifest.

One can, however, be interested in another problem, namely in the effective quantum action for the eigenvalues of the Polyakov line. Contrary to $A_{4}$ and to the Polyakov line as a unitary matrix, its eigenvalues are invariant under spatial gauge transformations. Therefore, the effective action for the eigenvalues can be expanded in ordinary 'short' rather than covariant derivatives, and the background $A_{i}$ field may then be set to zero. In this setting, the $A_{i}$ fields, as well as the rapidly changing components of the $A_{4}$ fields, are understood and treated as quantum fluctuations over which one integrates.

Fortunately, one does not need to solve this problem anew: in fact the result can be obtained from the more general case considered above. Assuming the gauge where $A_{4}$ is static and diagonal (such that the eigenvalues of the Polyakov line are given by eq. (29) expressed through $\nu_{1,2,3}$ ) all one practically needs to do is to set $A_{i}$ to zero, which means putting to zero all components of the magnetic field $B_{i}$ and the non-diagonal components of the electric field $E_{i}^{1,2,4,5,6,7}$. What is left are the $E_{i}^{3,8}$ components of the electric field whose definition is $E_{i}^{3,8}=\partial_{i} A_{4}^{3,8}+f^{(3,8) c(3,8)} A_{i}^{c} A_{4}^{3,8}=\partial_{i} A_{4}^{3,8}$, i.e. they are independent of $A_{i}$. One can easily check that these components correspond to combinations of the invariants (6364) in which $A_{i}$ is canceled, even if one does not assume $A_{4}$ to be diagonal. We remark that our coefficient functions of $A_{4}$ in front of $E_{i}^{3,8}$ coincide with those computed recently in ref. [21].

In terms of the quantities $\nu_{1,2,3}(x)$ the diagonal components of the electric field are, according to eq. (35),

$$
E_{i}^{3}=\partial_{i} \nu_{1} 2 \pi T, \quad E_{i}^{8}=\frac{\partial_{i}\left(\nu_{2}-\nu_{3}\right)}{\sqrt{3}} 2 \pi T .
$$

The last line in eq. (42) gives the needed two-derivative terms in the gauge-invariant effective action for the eigenvalues of the Polyakov line. Assembling terms proportional to $h\left(\nu_{1}\right), h\left(\nu_{2}\right), h\left(\nu_{3}\right)$ and using $\nu_{1}=\nu_{2}+\nu_{3}$ we get a remarkably symmetric expression:

$$
\begin{aligned}
S^{\mathrm{kin}} & =\int d^{3} x T \frac{11}{12}\left\{-2 \log \left(\frac{4 \pi T}{\Lambda e^{\gamma_{E}}}\right)\right. \\
& \times\left[\left(\partial_{i} \nu_{1}\right)^{2}+\left(\partial_{i} \nu_{2}\right)^{2}+\left(\partial_{i} \nu_{3}\right)^{2}\right] \\
& \left.+\left[\left(\partial_{i} \nu_{1}\right)^{2} H\left(\nu_{1}\right)+\left(\partial_{i} \nu_{2}\right)^{2} H\left(\nu_{2}\right)+\left(\partial_{i} \nu_{3}\right)^{2} H\left(\nu_{3}\right)\right]\right\},
\end{aligned}
$$

where

$$
H(\nu)=\left[-\psi(\nu)-\psi(1-\nu)-2 \gamma_{E}\right]_{\bmod 1},
$$

and the eigenvalues of the Polyakov loop in terms of $\nu_{1,2,3}(x)$ are given by eq. (29). Since the $Z(3)$ symmetry consists in shifting $\nu_{1,2,3}$ (i.e. the eigenvalues of $A_{4}$ in the adjoint representation) by integers, and the function $H(\nu)$ is periodic with period 1 , this action is explicitly 
$Z(3)$ symmetric. It should be reminded that $\nu_{1,2,3}$ are not independent but satisfy $\nu_{1}=\nu_{2}+\nu_{3}$, see eq. (35). The renormalization has been performed in the PauliVillars scheme. If another, e.g. the Modified Minimal Subtraction $(\overline{\mathrm{MS}})$ scheme is used, one has to replace $\Lambda$ by $e^{\frac{1}{22}} \Lambda_{\overline{\mathrm{MS}}}[23]$.

The function $H(\nu)$ (which appears also in the $S U(2)$ case [13, 17]) is plotted in Fig[2] It goes as $\frac{1}{\nu}$ or as $\frac{1}{1-\nu}$ as $\nu$ approaches 0 or 1 , respectively. The singularity is due to the contribution of the zero Matsubara frequency, i.e. of the static quantum fluctuations. One may wish to disregard this contribution but then the periodicity of $H(\nu)$ is lost, together with the $Z(3)$ symmetry of the action.

Eq. (86) together with eq. (34) for the potential energy is the $S U$ (3) effective action for the eigenvalues of the Polyakov line (29), up to two spatial derivatives in the 1-loop order. It is interesting that both the 'potential' and 'kinetic' energy parts are sums over eigenvalues of $A_{4}$ in the adjoint representation.

\section{B. General SU(N) case}

As before, it is possible and convenient to choose the gauge such that $A_{4}$ is static and diagonal in the fundamental representation:

$$
\begin{aligned}
A_{4}(x)= & A_{4}^{a} t^{a}=2 \pi T \operatorname{diag}\left(a_{1}, a_{2}, \ldots, a_{N}\right), \\
& a_{1}+a_{2}+\ldots+a_{N}=0,
\end{aligned}
$$

where the common factor $2 \pi T$ is taken for future convenience. The Polyakov line is then a diagonal $S U(N)$ unitary matrix,

$$
L(x)=\operatorname{diag}\left(e^{2 \pi i a_{1}}, e^{2 \pi i a_{2}}, \ldots, e^{2 \pi i a_{N}}\right) .
$$

The effective action must be invariant under timedependent gauge transformations, generalizing eq. (8):

$$
\begin{aligned}
A_{4} \rightarrow & S A_{4} S^{\dagger}+i S \partial_{t} S^{\dagger} \\
S= & \operatorname{diag}\left(e^{i\left[2 \pi t T \mu_{1}+\alpha_{1}(x)\right]}, \ldots, e^{i\left[2 \pi t T \mu_{N}+\alpha_{N}(x)\right]}\right) \\
& \sum_{m=1}^{N} \mu_{m}=0, \quad \sum_{m=1}^{N} \alpha_{m}(x)=0
\end{aligned}
$$

This gauge transformation amounts to shifting $a_{m} \rightarrow$ $a_{m}+\mu_{m}$. The time frequencies $\mu_{m}$ are quantized because this gauge transformation written down for the adjoint representation must be periodic in time, meaning that $O^{a c}(t=0) O^{b c}(t=1 / T)=\delta^{a b}$ where $O^{a b}=2 \operatorname{Tr}\left(S t^{a} S^{\dagger} t^{b}\right)$, $\operatorname{Tr} t^{a} t^{b}=\frac{1}{2} \delta^{a b}$. This periodicity in time leads to the requirement that all differences

$$
\mu_{m}-\mu_{n}=\text { integers. }
$$

Given the condition (92), the general solution to eq. (93) is

$$
\mu_{m}=\frac{k_{0}}{N}+k_{m}, \quad \sum_{m=0}^{N} k_{m}=0
$$

where $k_{0}, k_{1}, \ldots, k_{N}$ are all integer numbers. Under the gauge transformation (91) the Polyakov line is multiplied by a diagonal matrix

$$
S^{\dagger}(0) S(1 / T)=\operatorname{diag}\left(e^{2 \pi i \mu_{1}}, \ldots, e^{2 \pi i \mu_{N}}\right) \in Z(N)
$$

which belongs to the group center when $\mu$ 's are given by eq. (94).

It follows from the general formulae of section II, that the effective action is a functional of the eigenvalues of $A_{4}$ in the adjoint representation. If $A_{4}$ in the fundamental representation is diagonal and given by eq. (88), the eigenvalues of the adjoint $\left(N^{2}-1\right) \times\left(N^{2}-1\right)$ matrix $A_{4 \text { adj }}^{a b}=i f^{a c b} A_{4}^{c}$ are

$$
\text { adjoint eigenvalues }= \pm 2 \pi T\left(a_{m}-a_{n}\right) \equiv \pm 2 \pi T \nu_{m n} .
$$

There are $(N-1)$ zero eigenvalues whose number is the rank of the group, and $N(N-1)$ pair-wise $( \pm)$ non-zero eigenvalues. Under gauge transformation (91) the adjoint eigenvalues apparently shift by integers,

$$
\nu_{m n}=\left(a_{m}-a_{n}\right) \rightarrow \nu_{m n}+\left(\mu_{m}-\mu_{n}\right)=\nu_{m n}+\text { integers }
$$

owing to eq. (93). Therefore, the $Z(N)$ symmetry of the effective action is manifest if it is periodic in adjoint eigenvalues $\nu_{m n}$ with period 1 .

With $A_{i}$ set to zero, the calculation of the invariants (39.41) simplifies. Basically, they are sums of functionals of the adjoint eigenvalues $\nu_{m n}$. The tree-level kinetic energy $E_{i}^{a} E_{i}^{a} / 2=\operatorname{Tr}\left(\partial_{i} A_{4}\right)^{2}=(2 \pi T)^{2} \sum_{m=1}^{N}\left(\partial_{i} a_{m}\right)^{2}$ can be also written as a sum over adjoint eigenvalues, using the identity

$$
\sum_{m=1}^{N}\left(\partial_{i} a_{m}\right)^{2}=\frac{1}{N} \sum_{m<n}^{N}\left[\partial_{i}\left(a_{m}-a_{n}\right)\right]^{2},
$$

which is satisfied when $\sum_{1}^{N} a_{n}=0$ is taken into account. We thus obtain the following 1-loop effective action for the eigenvalues of the Polyakov loop in a general $S U(N)$ theory:

$$
\begin{aligned}
S= & -\sum_{m>n}^{N} \int d^{3} x \\
& \times\left\{\left(\partial_{i} \nu_{m n}\right)^{2} \frac{11}{12} T\left[2 \log \left(\frac{4 \pi T}{\Lambda e^{\gamma_{E}}}\right)-H\left(\nu_{m n}\right)\right]\right. \\
& \left.+\frac{(2 \pi)^{2} T^{3}}{3} \nu_{m n}^{2}\left(1-\nu_{m n}\right)^{2}\right\}, \quad \nu_{m n}=a_{m}-a_{n},
\end{aligned}
$$

where the function $H(\nu)$ is given by eq. (87) and plotted in Fig. 2: it is periodic with period 1. There are $N(N-1) / 2$ similar terms in eq. (99), however it should be kept in mind that there are only $N-1$ independent variables $a_{m}(x)$ through which the eigenvalues of the Polyakov line are expressed. At $N=3$ there are three terms and the general result (99) comes to eq. (86). At $N=2$ there is only one term, and the result coincides with that of refs. [13, 17]. The minima of 
the potential energy lie at integer values of $\nu_{m n}$ corresponding to the Polyakov line belonging to the group center. The maxima correspond to the Polyakov line $L_{\max }=\operatorname{diag}\left(e^{i \pi \frac{N-1}{N}}, e^{i \pi \frac{N-3}{N}}, \ldots, e^{-i \pi \frac{N-1}{N}}\right)$ (and those which one gets from this one by multiplying it by elements of the center), and it has the property that $\operatorname{Tr} L_{\max }=0$. It is interesting that $H(\nu)$ is positive definite, so that the kinetic energy changes sign at certain values of $\nu$ depending on the temperature. It may signal an instability of the trivial (perturbative) vacua, as one lowers the temperature.

\section{CONCLUSIONS}

We derived an effective 1-loop action for the Polyakov line in a pure Yang-Mills theory in two settings: 1) for the Polyakov line as a unitary matrix rotating under spatial gauge transformations, 2) for the gauge-invariant eigenvalues of the Polyakov line. We do not assume that the Polyakov line is close to the elements of the center, meaning that we are collecting all powers of the $A_{4}$ field in the effective action while expanding in its (covariant) derivatives.

In case 2 the effective action is an expansion in ordinary spatial derivatives of the Polyakov line eigenvalues, and is explicitly symmetric under discrete $Z(N)$ transformations of the eigenvalues. In case 1 the effective action is an expansion in covariant derivatives of $A_{4}$, which necessarily include the spatial components $A_{i}$ of the background field. We expand the action to include all invariants of the type $E_{i}^{2}$ where $E_{i}$ is the electric field and of the type $B_{i \|}^{2}$ where $B_{i}$ is the magnetic field parallel, in the $\mathrm{SU}(3)$ sense, to $A_{4}$. We have checked our results by reducing them to the previously studied $S U(2)$ case, and by comparing them with the previously known expansion up to quadratic terms in $A_{4}$. We stress, however, that we collect all powers of $A_{4}$ in our effective action.

In case 1 our results are symmetric with respect to the group center only in part of the gauge invariants we compute but not in all of them. The reason is that the $Z(N)$ symmetry is actually a consequence of the invariance under fast time-dependent gauge transformations. Once $A_{i}$ fields are introduced to ensure gauge invariance of the effective action under static transformations of the Polyakov line, the time-dependent transformations generate large time derivatives of $A_{i}$. Unless all powers in $\dot{A}_{i}$ are collected in the effective action, the $Z(N)$ symmetry is not manifest. Since we expand to the order of $E^{2}$ only (hence to the second order in $\dot{A}_{i}$ ) we cannot observe the $Z(N)$ symmetry. Our results in case 1 apply to static $\left(A_{i}, A_{4}\right)$ background fields and to the $S U(3)$ group only.

We hope that the results may be of some help to study correlation functions of the Polyakov line not too far from the transition point where it experiences fluctuations that are large in amplitude but presumably mainly long ranged.

\section{APPENDIX A: BASICS ABOUT $S U(3)$}

The generators of the gauge group $S U(3)$ are

$$
t^{a}=\frac{\lambda^{a}}{2}
$$

where the matrices $\lambda^{a}$ are given by

$$
\begin{aligned}
\lambda^{1} & =\left(\begin{array}{lll}
0 & 1 & 0 \\
1 & 0 & 0 \\
0 & 0 & 0
\end{array}\right), \lambda^{2}=\left(\begin{array}{ccc}
0 & -i & 0 \\
i & 0 & 0 \\
0 & 0 & 0
\end{array}\right), \\
\lambda^{3} & =\left(\begin{array}{ccc}
1 & 0 & 0 \\
0 & -1 & 0 \\
0 & 0 & 0
\end{array}\right), \lambda^{4}=\left(\begin{array}{lll}
0 & 0 & 1 \\
0 & 0 & 0 \\
1 & 0 & 0
\end{array}\right), \\
\lambda^{5} & =\left(\begin{array}{ccc}
0 & 0 & -i \\
0 & 0 & 0 \\
i & 0 & 0
\end{array}\right), \lambda^{6}=\left(\begin{array}{lll}
0 & 0 & 0 \\
0 & 0 & 1 \\
0 & 1 & 0
\end{array}\right), \\
\lambda^{7} & =\left(\begin{array}{ccc}
0 & 0 & 0 \\
0 & 0 & -i \\
0 & i & 0
\end{array}\right), \lambda^{8}=\frac{1}{\sqrt{3}}\left(\begin{array}{ccc}
1 & 0 & 0 \\
0 & 1 & 0 \\
0 & 0 & -2
\end{array}\right) .
\end{aligned}
$$

The generators satisfy

$$
\left[t^{a}, t^{b}\right]=i f^{a b c} t^{c}
$$

In addition to the totally anti-symmetric $f^{a b c}$ there are totally symmetric $d^{a b c}$ structure constants which are defined according to

$$
\left\{t^{a}, t^{b}\right\}=\frac{1}{3} \delta^{a b}+d^{a b c} t^{c} .
$$

The non-vanishing values of $f^{a b c}$ and $d^{a b c}$ are summarized in Table A5

\begin{tabular}{rr|rr}
\hline \hline$(\mathrm{a}, \mathrm{b}, \mathrm{c})$ & $f^{a b c}$ & $(\mathrm{a}, \mathrm{b}, \mathrm{c})$ & $d^{a b c}$ \\
\hline \hline 123 & 1 & 118 & $1 / \sqrt{3}$ \\
147 & $1 / 2$ & 146 & $1 / 2$ \\
156 & $-1 / 2$ & 157 & $1 / 2$ \\
246 & $1 / 2$ & 228 & $1 / \sqrt{3}$ \\
257 & $1 / 2$ & 247 & $-1 / 2$ \\
345 & $1 / 2$ & 256 & $1 / 2$ \\
367 & $-1 / 2$ & 338 & $1 / \sqrt{3}$ \\
458 & $\sqrt{3} / 2$ & 344 & $1 / 2$ \\
678 & $\sqrt{3} / 2$ & 355 & $1 / 2$ \\
& & 366 & $-1 / 2$ \\
& & 377 & $-1 / 2$ \\
& & 448 & $-1 /(2 \sqrt{3})$ \\
& & 558 & $-1 /(2 \sqrt{3})$ \\
& & 668 & $-1 /(2 \sqrt{3})$ \\
& & 778 & $-1 /(2 \sqrt{3})$ \\
\hline \hline
\end{tabular}


The $t^{a}$ matrices fulfill the following relations

$$
\begin{aligned}
t_{i j}^{a} t_{k l}^{a} & =\frac{1}{2}\left[\delta_{i l} \delta_{j k}-\frac{1}{3} \delta_{i j} \delta_{k l}\right], \\
\operatorname{Tr} t^{a} & =0 \\
\operatorname{Tr}\left(t^{a} t^{b}\right) & =\frac{\delta^{a b}}{2} \\
\operatorname{Tr}\left(t^{a} t^{b} t^{c}\right) & =\frac{1}{4}\left(d^{a b c}+i f^{a b c}\right), \\
\operatorname{Tr}\left(t^{a} t^{b} t^{a} t^{c}\right) & =-\frac{1}{12} \delta^{b c} .
\end{aligned}
$$

The structure constants satisfy the Jacobi identities:

$$
\begin{gathered}
f^{a b e} f^{e c d}+f^{c b e} f^{a e d}+f^{d b e} f^{a c e}=0 \\
f^{a b e} d^{e c d}+f^{c b e} d^{a e d}+f^{d b e} d^{a c e}=0 .
\end{gathered}
$$

Additionally

$$
f^{a b e} f^{c d e}=\frac{2}{3}\left(\delta^{a c} \delta^{b d}-\delta^{a d} \delta^{b c}\right)+\left(d^{a c e} d^{b d e}-d^{b c e} d^{a d e}\right) .
$$

By defining the $8 \times 8$ matrices $F^{a}$ and $D^{a}$ such that

$$
\begin{aligned}
& \left(F^{a}\right)^{b c}=-i f^{a b c} \\
& \left(D^{a}\right)^{b c}=d^{a b c}
\end{aligned}
$$

the Jacobi identities A11A12 take the form

$$
\begin{aligned}
& {\left[F^{a}, F^{b}\right]=i f^{a b c} F^{c},} \\
& {\left[F^{a}, D^{b}\right]=i f^{a b c} D^{c} .}
\end{aligned}
$$

And the fact that $f^{a b b}=0$ and $d^{a b b}=0$ implies

$$
\operatorname{Tr} F^{a}=0 \quad, \quad \operatorname{Tr} D^{a}=0 .
$$

Some more useful relationships are:

$$
\begin{aligned}
f^{a c d} f^{b c d} & =3 \delta^{a b}, \\
F^{a} F^{a} & =3, \\
\operatorname{Tr}\left(F^{a} F^{b}\right) & =3 \delta^{a b}, \\
f^{a c d} d^{b c d} & =0, \\
F^{a} D^{a} & =0 \\
\operatorname{Tr}\left(F^{a} D^{b}\right) & =0 \\
d^{a c d} d^{b c d} & =\frac{5}{3} \delta^{a b}, \\
D^{a} D^{a} & =\frac{5}{3} \\
\operatorname{Tr}\left(D^{a} D^{b}\right) & =\frac{5}{3} \delta^{a b}, \\
\operatorname{Tr}\left(F^{a} F^{b} F^{c}\right) & =i \frac{3}{2} f^{a b c}, \\
\operatorname{Tr}\left(D^{a} F^{b} F^{c}\right) & =\frac{3}{2} d^{a b c}, \\
\operatorname{Tr}\left(D^{a} D^{b} F^{c}\right) & =i \frac{5}{6} f^{a b c}, \\
\operatorname{Tr}\left(D^{a} D^{b} D^{c}\right) & =-\frac{1}{2} d^{a b c}, \\
\operatorname{Tr}\left(F^{a} F^{b} F^{a} F^{c}\right) & =\frac{9}{2} \delta^{b c} . \\
\operatorname{Tan} & =f^{a}
\end{aligned}
$$

In particular for any matrix in the fundamental representation

$$
A=A^{a} t^{a}
$$

one can construct the adjoint representation according to

$$
A^{a b}=i f^{a c b} A^{c} \text {. }
$$

[1] T. Appelquist and R.D. Pisarski, Phys. Rev. D 23, 2305 (1981); S. Nadkarni, Phys. Rev. D 27, 917 (1983); ibid. 38, 3287 (1988); N.P. Landsman, Nucl. Phys. B 322, 498 (1989).

[2] A. M. Polyakov, Phys. Lett. B 72, 477 (1978).

[3] A. D. Linde, Phys. Lett. B 96, 289 (1980).

[4] D. J. Gross, R. D. Pisarski and L. G. Yaffe, Rev. Mod. Phys.53, 43 (1981).

[5] P. Arnold and C. Zhai, Phys. Rev. D 50, 7603 (1994); ibid. 51, 1906 (1995); E. Braaten, Phys. Rev. Lett. 74, 2164 (1995); B. Kastening and C. Zhai, Phys. Rev. D 52, 7232 (1995); E. Braaten and A. Nieto, Phys. Rev. D 53, 3421 (1996).

[6] L. Susskind, Phys. Rev. D 20, 2610 (1979).

[7] G.'t Hooft, Nucl. Phys. B 138, 1 (1978); ibid. 153, 141 (1979).

[8] B. Svetitsky and L. G. Yaffe, Nucl. Phys. B 210, 423 (1982).

[9] O. Kaczmarek, S. Ejiri, F. Karsch, E. Laermann and F. Zantow. Talk given at Workshop on Finite Density QCD at Nara, Japan, 2003. hep-lat/0312015
[10] A. Dumitru, Y. Hatta, J. Lenaghan, K. Orginos and R. D. Pisarski, Phys. Rev. D (to be published), hep-th/0311223

[11] N. Weiss, Phys. Rev. D 24, 475 (1981); ibid. 25, 2667 (1982).

[12] V.M. Belyaev and V.L. Eletsky, JETP Lett. 50, 55 (1989), Pisma Zh. Eksp. Teor. Fiz. 50, 49 (1989); K. Enqvist and K. Kajantie, Z. Phys. C 47, 291 (1990).

[13] D. Diakonov and M. Oswald, Phys. Rev. D 68, 025012 (2003).

[14] M. Oswald, Acta Phys. Pol. B 34, 5847 (2003).

[15] D. Diakonov and M. Oswald, Phys. Rev. D (to be published) hep-ph/0312126

[16] D. Diakonov, V. Yu. Petrov and A. V. Yung, Phys. Lett. B 130, 385 (1983); Yad. Fiz. 39, 240 (1984); Sov. J. Nucl. Phys. 39, 150 (1984).

[17] T. Bhattacharya, A. Gocksch, C. Korthals Altes and R.D. Pisarski, Nucl. Phys. B 383 (1992) 497.

[18] J. Schwinger, Phys.Rev.82, 664 (1951).

[19] S. Huang and M. Lissia, Nucl. Phys. B 438, 54 (1995); K. Kajantie, M. Laine, K. Rummukainen and M. E. Sha- 
poshnikov, Nucl. Phys. B 458, 90 (1996); ibid. 503, 357 (1997).

[20] S. Chapman, Phys. Rev. D 50, 5308 (1994).

[21] E. Megias, E. Ruiz Arriola and L. L. Salcedo, Phys. Rev. D (to be published), hep-ph/0312133.

[22] E. Megias, E. Ruiz Arriola and L. L. Salcedo, Phys. Lett.
B 563, 173 (2003).

[23] A. Hasenfratz and P. Hasenfratz, Phys. Lett. B 93, 165 (1980), Nucl. Phys. B 193, 210 (1981).

[24] Our convention for the sign of the action is such that the partition function $\mathcal{Z}=\exp (+S)$. 